\title{
TESTS OF SOCIAL DOMINANCE ON CHARITABLE INTENT TOWARDS MINORITIES
}

\section{BY}

\section{ZACCHAEUS VINCENT KEVIN BASTION}

\author{
A thesis submitted to Victoria University of \\ Wellington in fulfillment of the requirements for the \\ degree of Masters in Science (Psychology).
}

Victoria University of Wellington 2016 


\begin{abstract}
This thesis investigates the hypothesis that the design of adverts for charitable causes may be unintentionally priming viewers in Social Dominance Orientation (SDO: Pratto, Sidanius, Stallworth, \& Malle, 1994). Huang and Liu (2005) found evidence that presenting people with material that makes social hierarchies salient can temporarily increase a person's level of SDO. As many charities make differences in social hierarchy salient as part of their advertisement campaigns, it is possible that such advertisements may unintentionally prime the SDO of viewers. Given prior research that indicates that high SDO is correlated with antisocial attitudes (Sibley \& Duckitt, 2010), such priming may be reducing the likelihood that viewers would donate towards the charitable cause. First, a pre-test was conducted to test the psychometric structure and reliability of the $\mathrm{SDO}_{7}$ (Ho, et al., in print) and the Counter Dominance Orientation measure (CDO: Pratto et al., 2012). Confirmatory factor analysis of each measure found a four-factor model of SDO and a two-factor model of CDO. Subsequently, 139 firstyear students of psychology were used to test the previously-mentioned hypothesis. Participants were asked to critically evaluate one of two adverts for a student support service, with the one in the experimental condition being for a service for Māori and Pacifica students. The intent of this advert was to prime viewers on SDO. Afterwards, they engaged in a hypothetical public goods game where a portion of the money pool would go towards the service the advert was for. If the hypothesis was correct, participants who were primed on SDO would be less willing to contribute towards a cause that supported minorities, but the data did not support this. Implications and potential future research, both for the new measures and the hypothesis are explored.
\end{abstract}




\section{Tests of Social Dominance on Charitable Intent towards Minorities}

'Charity,' according to the definition relating to an organization, is an organization that solicits funds from the public to put towards a cause (Reiling, 1958). Thus, to gather these funds, charities must ask the public for donations. Priming people with images or narratives of preventable suffering is the most common means of soliciting charity, typically via advertisements on television and other media. For example, charities operating in third-world nations will typically show scenes of the extreme poverty and lack of resources that first-world nations take for granted. Examples include recent campaigns by multiple organizations, including the World Health Organization, the United Nations Children's Fund (UNICEF) and Médecins Sans Frontières to battle the 2014 Ebola virus outbreak in West Africa. The goal of such advertisements is, obviously, to inform the viewer about the need for donations. However, is there a risk that traditional campaign frames may actually reduce charitable giving by at least some potential donors? To use the previous example, might it be the case that by showing someone in a first-world nation the difficulties faced in thirdworld nations, potential first-world donors become more aware of the higher position in a social hierarchy they hold. Given the preference of post-industrial societies to develop belief systems and structures to perpetuate those hierarchies, might potential donors become less supportive of actions that would help equalize that hierarchy?

The aims of this research are to test two new measures of Social Dominance Orientation (SDO: a measure of the extent to which people endorse social hierarchy), as well as test the effects of advertisements similar to ones used by charities on donation behaviour. We begin with an in-depth examination of SDO, the factors that can influence it, and the factors that it in turn influences. We will then expand into altruism and how social dominance can influence it. From past research, we extrapolate that the traditional framing used by charitable organisations (and other organisations working to address health and social crises) may be paradoxically self-defeating. Specifically, the traditional method of soliciting donations (through images and narratives of outgroup disadvantage) may 'activate' potential donors existing hierarchical predispositions (SDO) and render them less likely to donate. 


\section{Social Dominance Theory}

SDO (Pratto, Sidanius, Stallworth, \& Malle, 1994) is considered the individual expression of a preference for social hierarchy that plays a central role in testing hypotheses derived from Social Dominance Theory (SDT: Sidanius \& Pratto, 1999). Sidanius and Pratto (1999) point out that society tend to be built around group-based social hierarchies with one, or some, dominant groups and a greater amount of subordinate groups. Dominant groups typically display a large amount of positive social value, a term that incorporates both material and abstract resources that society members aspire to. These may include economic (in the form of higher incomes and greater collective wealth) and political influence. Meanwhile, subordinate groups typically evidence greater negative social value. This typically includes markers such as low social standing, fewer opportunities in all areas, and increased likelihood of experiencing negative sanctions (for example, imprisonment for crime for which a dominant group member would receive a lesser punishment).

These hierarchies tend to come in three types: age-based, gender-based and arbitrary set-based (which includes the like of sexuality, religion, race and other miscellaneous, highly salient categories created by a society: Sidanius \& Pratto, 1999). Most societies have hierarchies of all three of these types. For example, we live in an age-based hierarchy, since adults have more power and privileges compared to children and teenagers. In addition, we live in a sex-based hierarchy, as men typically have more power and privilege compared to women (Berdahl, 2007; Carli, 1999; Pratto, Stallworth, \& Sidanius, 1997). Compared to age-based hierarchies, people's roles in these hierarchies are, for the most part, fixed. Meanwhile, arbitrary set-based hierarchies are just as resilient and unchanging as the previous two forms, despite being utterly arbitrary, where arbitrariness is defined in terms of whether or not the difference between groups has any grounding in physical reality. For example, race is an arbitraryset hierarchy because, at the genetic level, there is very little difference between humans of different races. Other differences, such as wealth, are entirely manmade, and so are arbitrary. Some of the most visible examples of such arbitrary hierarchies in modern society are race-based hierarchies. Societies typically have one or more races in a dominant position to which other racial groups are subordinate. Examples include white Americans being dominant over all other races in the United States, and Pākehā (a common term describing New Zealanders of European descent) being dominant over 
Māori in New Zealand. As different races of humans are the same in many respects other than superficial appearances, any subcategory of humans is constructed by humans. As it has no fundamental basis in physical reality, it is arbitrary.

SDT makes three assumptions (Sidanius \& Pratto, 1999). The first is that age and sex-based structures will exist in all societies, while arbitrary-set based hierarchies will only exist in societies capable of economic surplus. The second is that the root of most conflicts arises because of the existence of some form of social hierarchies. This assumption holds that all forms of discrimination, prejudice and conflict that spring from arbitrary set-based hierarchies such as racism, sexism, and homophobia, simultaneously produce and reflect the group-based social hierarchies that make up society. The third assumption is the existence of hierarchy-enhancing (HE) forces, which perpetuate social inequality, and hierarchy-attenuating (HA) forces, which promote more social equality (Sidanius \& Pratto, 1999). An example of a hierarchyenhancing institution would be the police and other internal security branches. As they grant a level of power and authority over most other groups, they become more endorsing of SDO (Sidanius, Liu, Shaw, \& Pratto, 1994).An example of hierarchyattenuating forces would be movements to institute equal rights between minorities and majorities. For example, consider the African-American Civil Rights Movement in the United States. The goal of this movement was to undo segregation between black people and white people, thus giving them social and economic equality (Weisbrot, 1990).

\section{Social Dominance Orientation}

To test these assumptions, Pratto, Sidanius, Stallworth, and Malle. (1994; see also Sidanius \& Pratto, 1999) developed a measure of Social Dominance Orientation. The SDO scale assesses endorsement of societal inequality, of hierarchies within a civilisation in which groups at the top of the hierarchy dominate groups below them in the hierarchy. SDO influences social and political attitudes, with those who score highly displaying more of a 'dog-eat-dog' view of the world; where one must sometimes push others down to improve one's own livelihood (Levin, Federico, Sidanius, \& Rabinowitz, 2002).

Politically, those who score high in SDO are more likely to hold right-wing or conservative views (Pratto, Sidanius, Stallworth, \& Malle, 1994). As such, they are 
more likely to vote for parties like the Republicans in North America (Pratto et al., 1994) and the National Party in New Zealand (Wilson \& Sibley, 2013). SDO reflects a general orientation (rather than an attitude specifically related to politics), so it can pertain to any group distinction that comes up in particular social contexts. Thus, SDO can influence attitudes in terms of physical group distinctions such as sex and age, as well as socially created distinctions, such as political or religious allegiance, or geographic and national differences.

SDO is argued to have a huge impact on both the intensity, and the nature, of group-based social hierarchy for two reasons. The first is that it seems to influence all social ideologies and legitimising myths, which are beliefs and stereotypes that make social hierarchies seem more desirable (Chen \& Tyler, 2001). The second, and most important point, is that it influences the output of both hierarchy-enhancing and hierarchy-attenuating social policies (Pratto et al. 1994) (Pratto, Sidanius, Stallworth, \& Malle, 1994). The scope of SDO is very broad, as it relates to individual attitudes, as well as large-scale issues like policy relating to the distribution of social value between groups (Pratto et al., 1994). (Pratto, Sidanius, Stallworth, \& Malle, 1994) Social value can include material things like wealth, resources and employment opportunities, as well as abstract values like power and status.

SDO is influenced by four factors. The first is the individual's membership in, and identification with, hierarchical groups that are both arbitrary and salient. Typically, one would find that people in dominant groups, or those who identify strongly with dominant groups, exhibit higher SDO than members of subordinate groups, or those who identify strongly with such groups (Sidanius, Pratto, \& Mitchell, 1993). Secondly, an individual's level of SDO is influenced by a multitude of background factors which can include the amount of education they have received, their religious beliefs and their social experiences (Sidanius, Pratto, \& Bobo, 1994). The third factor seems to be some kind of temperamental predisposition towards SDO. This may include a genetic factor, not necessarily for SDO, but for related factors such as empathy (Nishimura et al., 2004). There is evidence to believe that increased levels of empathy can lead to reduced levels of SDO (Sidanius, et al., 2013). The fourth factor, as mentioned later in this paper, is sex. Men typically show greater SDO than women (Sidanius \& Pratto, 1999). 
Tests of Social Dominance on Charitable Intent Towards Minorities

\section{Factors that influence Social Dominance Orientation}

Sex

There are individual differences in SDO, with those in different demographics showing different levels of SDO. On average, men show higher SDO than women (Pratto, Stallworth, \& Sidanius, 1997). This is called the invariance hypothesis. It has been speculated that hormones that vary in levels between sexes, such as testosterone, play a role in this (Sellers, Mehl, \& Josephs, 2007). Another possibility is that one's position in a social hierarchy plays a role in how much SDO you show (Sidanius, Liu, Shaw, \& Pratto, 1994). As an example, in Western society, men typically hold the higher position in the sex-based hierarchy. As such, because they enjoy the benefit of being higher in a hierarchy, they endorse such hierarchies more. Sidanius, Pratto, and Bobo (1994) provided evidence that men tend to show greater SDO than woman across factors such as culture, demographics and a wide variety of situational factors. Additionally, police officers tend to have higher SDO than the general population, as do others occupying hierarchy-enhancing social roles (such as military, prosecuting lawyers, financial market workers, and others: Sidanius, Pratto, Sinclair, \& van Laar, 1996)

However, there is evidence that it is more than biological sex that influences SDO. For example, Wilson and Liu (2003) looked at the effects of gender identity on SDO, reporting evidence that the level of identification one feels with their sex moderates SDO. As such, strength of gender identification correlates positively with SDO in men, and negatively in women. These findings imply that traditionally 'masculine' traits play a role in increasing SDO.

If SDO varies so strongly between sexes, does that influence other differences observed between sexes? Pratto et al. (1997) considered the influence of SDO on political allegiances between genders. They reported evidence supporting the idea that men support conservative political parties and ideologies more. Women, however, are more likely to support equal rights and social programmes. They also found evidence for SDO accounting for a large amount of this variation between political attitudes among sexes. 
Race

Racial groups may be considered arbitrary-set groups. Do minorities typically show less SDO compared to the majorities who have a position of power in a social hierarchy? Sidanius, Levin, Liu, and Pratto (2000) report evidence for both the cultural deterministic and social deterministic paradigms of SDO. Drawing on multiple international samples, they found evidence that defining social status in different ways, such as wealth or race result in differences in levels of anti-egalitarianism between members of dominant and subordinate groups based on a variety of situational and contextual factors. Again, men showed greater SDO than women across all cultures measured.

Additionally, Sidanius, Liu, Shaw, and Pratto (1994) also report evidence from a USA sample that those who are higher in social hierarchies show greater SDO: police reported higher SDO than jurors, who in turn were higher than public defenders; EuroAmericans were higher in SDO than African Americans or Hispanics. They also found evidence that these factors may interact, as Euro-American police officers reported the highest levels of SDO of all tested groups. Thus, it is clear that SDO influences outgroup attitudes, but can it also influence ingroup attitudes?

\section{Ingroup-outgroup attitudes and SDO}

Sidanius et al. (1994) investigated the degree to which people assign virtues to their ingroups based on legitimacy of the group, self-esteem, sex and SDO. Results suggested that the greater the SDO someone shows, the more virtuous they believe their ingroups are, even when other factors were accounted for. Similarly, Levin, Federico, Sidanius, and Rabinowitz (2002) hypothesized that favouritism towards high-status group was dependant on the social group one belonged to, and the perceived legitimacy of that high-status social group. Their theory was that, in high-status groups, SDO would be positively correlated with favouritism towards high-status groups, regardless of legitimacy of that group while low-status groups would show high-status group favouritism only when that high-status group was perceived as legitimate. To test these theories, they had Israeli Jews and Israeli Arabs complete measures of SDO, along with measures of perceived group status, perceived legitimacy of that status, and traits for each group. The results supported these hypotheses, with Israeli Arabs participants (a group low on the social hierarchy) showing more favouritism towards Israeli Jews (the 
group higher in the social hierarchy) when they have higher levels of SDO and view the Israeli Jews' position on the social hierarchy as legitimate. However, perceived legitimacy had no effect on how Israeli Jews perceived their own social status. A follow-up study generalized these results to the West, using Whites and Latinos in the United State. This suggests that this effect of SDO could be universal across cultures. Thus, it can be said that, across cultures, SDO impacts how we perceive both groups we belong to, and those we do not. Such perceptions and attitudes can shape how we behave towards them, either positively or negatively.

What is the exact mechanism through which SDO might influence the decision to support a minority or a group lower on the social hierarchy? Sibley and Duckitt, (2010) proposed that beliefs that involve treating people as individuals rather than as groups (such as meritocracy, the idea of judging people based on personal accomplishments) causes people to oppose social policies that would promote social equality by improving the livelihood of minorities, such as welfare programmes. Because such programmes can be interpreted as taking resources away from the dominant group, it is in their best interests to defend them. SDO then makes people believe different groups and demographics have differing levels of motivation and ambition. This highlights the role and importance of legitimizing myths. As people come to believe these myths, they begin to support hierarchy-enhancing policies and oppose hierarchy-attenuating policies, citing legitimizing myths as the reason.

These findings seem to suggest that a high level of SDO negatively impacts any personal opinions one might have of those who are socially 'beneath' them. As a result of this, they become less likely to help such people. Thus, we can reasonably assume that someone high in SDO would not behave in an altruistic way towards those of a lower social group. Now, we would like to turn our attention towards another variable that has been correlated with SDO in a variety of cases, Right-Wing Authoritarianism. It may be possible that it too, might impact how likely people are to charitably support others.

\section{Right-Wing Authoritarianism}

Right-Wing Authoritarianism (RWA) is assessed as the combination of three attitudinal facets that correlate together. The first is authoritarian submission, a measure of how willing someone is to submit to an authority they perceive as legitimate. The 
second is authoritarian aggression, a measure of how aggressive someone is towards those who deviate from societal norms or who oppose authority. The third is conventionalism, a measure of how much someone adheres to established societal norms and how much they feel others should do the same (Altemeyer, 1996). While SDO and RWA are typically found to correlate weakly (e.g., Pratto et al., 1994; Altemeyer, 1996) they make a unique contribution to predicting ingroup and outgroup attitudes (Altemeyer, 1998; Duckitt et al., 2002; Sibley, Robertson \& Wilson, 2006).

However, while SDO and RWA are commonly correlated, they are quite different constructs. As an example, consider the findings of Duckitt (2006). He investigated the effects of SDO and RWA on prejudice, looking at the pathways through which the two constructs seemed to make people more prone to prejudiced opinions. His findings imply that RWA is related to valuing social order and stability, and so it correlates with negative attitudes towards groups that are perceived as threatening the social order, such as deviant social groups. This includes drug dealers and rock stars, two such groups used in the study. SDO however, is related to valuing power and ingroup dominance other others. As such, it correlates with negative attitudes towards groups that are seen as socially subordinate, such as housewives, the physically disabled and those receiving unemployment benefits. These correlations were also found to be mediated by different factors. For RWA, their negative attitudes towards the socially deviant were mediated by the perceived threat of the group, while negative attitudes related to SDO were mediated by the perceived competitiveness of the groups. These findings suggest that while SDO and RWA may typically influence a broad factor such as prejudice in the same direction, the specific mechanics of how they do so can differ wildly.

Further evidence on the divergence of SDO and RWA comes from Heaven and Bucci (2001). They found that the two differed on how they correlated with Big Five personality constructs. RWA was positively correlated with conscientiousness, while SDO was negatively correlated with agreeableness. They also found that RWA had a link with dutifulness, as well as with orderliness and morality. They explained this as why those who score highly in RWA are so open to tradition and the stability that rigid authority brings. SDO, on the other hand, had negative correlations with duty, morality, cooperation and sympathy. This paints those high in SDO as someone who is unwilling 
to cooperate, unsympathetic towards others and low in morals. As such, it is clear that the two constructs are rooted in entirely different personality structures.

Building from Heaven and Bucci (2001), it seems that both factors are related to morality to some degree. SDO appears to have a negative correlation, contrasting with the positive correlation that RWA seems to have. Morality however, is an umbrella term. If it were broken down more into separate ethical positions, what would be found? Finding out was the goal of Wilson (2003). In his study, participants filled in measures of SDO and RWA, as well as the Ethics Position Questionnaire developed by Forsyth (1980). It measures endorsement of idealism (the belief that actions should cause no harm to others) as well as relativism (the belief that what is ethical is not set in stone, and shifts depending on circumstance). His findings implied a negative correlation between SDO and idealism, suggesting that those who score highly in SDO are more 'ruthless' in the pursuit of their goals, adopting a reasoning of 'the ends justify the means.' RWA was ultimately cut from the analysis, due to concerns over the possibility that those high in it would give a socially desirable answer, as opposed to a genuine one. However, past papers such as Altemeyer (1998) have found a correlation between RWA and idealism, so the possibility that such a relationship exists cannot be entirely discounted.

Going further into this vein of interaction between SDO, RWA and morality, the influence that SDO and RWA have on moral signatures also differs, according to Milojev et al. (2014). 'Moral signatures' refers to the degree to which an individual emphasizes the importance of the five moral foundations presented in the Moral Foundations Theory (MFT). MFT holds that morality is innate to some degree, but moulded by life experiences and culture. The five innate foundations of morality consist of care/harm, fairness/cheating, loyalty/betrayal, authority/subversion, and sanctity/degradation (Graham et al., 2012).

As mentioned, Milojev et al. (2014) found that SDO and RWA correlated with different moral signatures. In their study, they first aimed to identify a cluster of common moral signatures, and found four. High Moralists is when all five moral foundations are viewed as equally important, and corresponded with past research that identified a politically conservative moral cluster. Individiuators showed high value in the two individualizing foundations (care/harm and fairness/cheating, the two most 
focussed on the individual) but low value in the three binding foundations (loyalty/betrayal, authority/subversion and sanctity/degradation, the three most important for functioning in society). The third was Moderates, who showed average investment across all five foundations. The fourth signature, Neutral, did not match any previous political cluster, and was associated with low endorsement of all moral foundations. Furthermore, it was found that SDO was positively correlated with the Neutral signature, but negatively correlated with High Moralist and Individuator signature. RWA meanwhile, was positively correlated with the High Moralist signature, but negatively correlated with the Individuator and Neutral signature.

From this findings, we can assume that, if RWA does correlate negatively with charitable intent, it likely will not for the same reasons that SDO does. RWA has positive correlations with morality while SDO correlates more positively with an absence of morals. Thus, RWA would not correlate negatively with charitable intent due to an absence of morals, if at all.

The reason we wish to be clear that SDO and RWA are entirely separate variables that tend to correlate strongly is because the topic of this study revolves around a variable that RWA might not influence. One can easily make an argument for SDO having a negative correlation with charitable intent, since charities are examples of HA forces which those high in SDO are typically against (Sidanius \& Pratto, 1999). However, as mentioned, RWA is more opposed to deviant groups who threaten the social order (Duckitt, 2006), which does not typically include those who benefit from charity. Indeed, an argument could be made for the idea that RWA would have a positive correlation with charitable giving.

At this point, we have broached the topic of charity and altruism several times in this section. We now wish to cover the topic in more depth.

\footnotetext{
Altruism

Altruism is the expression of goodwill and charity without the expectation of a reward (Dictionary, 2015). Common acts of altruism include donating to charity, giving blood and volunteer work (Barclay, 2011). Altruism can spring from multiple sources, one of which is empathy (de Waal, 2008). Empathy can inspire altruism in multiple different ways. The first is through emotional cognition, where an individual has a
} 
negative emotional reaction to seeing others in distress resulting in an altruistic action that reduces the other's distress and thus, the individual's as well (Shamay-Tsoory, Aharon-Peretz, \& Perry, 2008). Another way that empathy can increase altruism is through sympathetic concern, which differs from emotional cognition in that it involves concern for others, rather than self-centred distress caused by the plight of others. It is this state of empathetic arousal that can drive people to life-threatening bravery to protect others from harm (Zahn-Waxler \& Radke-Yarrow, 1990). One more method is empathetic perspective-taking; in which one imagines themselves in a situation someone else is experiencing (de Waal, 2008). For our purposes, we can say that people donate to charity because they feel empathy for the plight of those the charity would benefit. As a result of this, they altruistically donate to the charity.

A common method of gauging altruistic behaviour in an experimental context is through public goods games. As an example of how public goods games can go, Fischbacher, Gächter and Fehr (2001) looked into how people might donate to public good games. In their study, they had participants make two decisions when it came to donating to a public goods pool. The first decision was an 'unconditional contribution,' in which they simply indicated how much they were willing to give. The second was a 'conditional contribution.' For this decision, participants were presented with a table that lists 21 possible average contribution levels from the other participants. For each of these averages, they had to indicate how much they would donate. In their results, they found that fifty percent of participants were conditionally cooperative. These participants in the conditional contribution section typically donated an amount similar to the averages. Thirty percent of participants were dubbed 'free-riders' as they displayed selfish attitudes by donating very little.

However, arguments have been made that altruism is not purely selfless (Mook, 1991). The most common claim is that the feeling you get for performing supposedly selfless deeds is its own reward. Such rewards, including the knowledge that you have done something good and the positive affect that gives, are called 'intrinsic' rewards. As such, because people are being rewarded for altruistic acts, they are technically not fully altruistic.

Studies have been performed to look at the neurobiological correlates of altruistic actions. Such studies provide evidence for the proposition that seemingly 
altruistic actions still benefit someone. For example, Moll et al. (2006) found that different types of charitable actions were correlated with activation in different parts of the brain. Monetary rewards and charitable donations both activated the mesolimbic reward pathway, the part of the brain that activates in response to pleasurable stimuli. However, charitable donations that genuinely placed others before oneself activated the subgenual cortex and the septal region of the brain. These sections are related to social bonding and attachment to others. These findings suggested that altruism is not the result of suppression of selfish urges, but a pleasurable activity that is built into the brain.

From a psychological perspective, there are issues relating to the definition of altruism. As mentioned earlier, some argue that true altruism is impossible when actions that could be called altruistic engage the mesolimbic reward pathway, making one feel good for performing supposedly altruistic actions (Moll, et al., 2006). To sidestep this, The International Encyclopaedia of the Social Sciences differentiates altruism and psychological altruism, and defines the latter as "a motivational state with the goal of increasing another's welfare" (pp. 87-88). However, there are still concerns that people are incapable of even psychological altruism. Because most acts of altruism can ultimately benefit the self in some way (improving social relations or standing with those one helps or just good feelings), such actions might not be completely selfless.

Is intent to donate to charity (a common example of a supposedly selfless action) truly the result of social forces, or do personal moral codes push us towards charitable behaviour? A study by van der Linden (2011) investigated this. The Theory of Planned Behaviour created by Ajzen (1991) states that people's intentions guide their behaviour, and they will carry out their intentions when they have enough control over their behaviour. A revised version of this theory was later presented by Smith and McSweeney (2007). While the original included three relevant constructs ('Attitude,' 'Perceived Behavioural Control,' and 'Descriptive Norms,') the revised model included 'Prescriptive Norms,' 'Moral Norms,' and 'Past Behaviour.' In van der Linden (2011)'s study, the revised model was used, each variable measured in the participants and correlated with willingness to donate to a charitable cause. Results indicated that moral norms were actually an incredibly powerful contributor to charitable behaviour, even greater than attitudes and perceived behavioural control. They also accounted for a large amount of the variance in intention to donate. This information is contrary to previous 
studies, which found that social pressures were the most prominent factor in people's decisions to donate to charitable causes. Now that we have given some background, we return to SDO, and look at how it might interact with altruism.

\section{Social Dominance and altruism}

D any evidence exist that even implies that altruism and SDO have any relationship at all? For example, is there any evidence that they may influence one variable in different ways? Zettler and Hilbig (2010) provide some evidence that this may be the case, in an investigation of the effects of altruism on political beliefs and opinions. They hypothesised that those who are highly altruistic would be more likely to support left-wing political ideologies (e.g., would support Democratic or Labour parties), and that altruism would explain variance in political allegiances independent of Big Six personality traits. Their results suggested a strong correlation between altruism and political orientation, one that persisted after variance caused by the Big Six personality factors was taken into account. Those who were more altruistic were more likely to support left-wing policies.

Zettler, Hilbig, and Haubrich (2011) provided further support, testing the effects of dispositional altruism on hypothetical and actual voting behaviour, as well as on endorsement of specific political agendas. Their results indicated that those who scored high on measures of altruism were more likely to vote for left-wing policies and parties, both in hypothetical scenarios and in reality. Given past research that indicates that those who score highly in measures of SDO were more likely to support right-wing parties and policies (Pratto, Sidanius, Stallworth, \& Malle, 1994) (Sibley \& Wilson, 2007), this is evidence for the idea that altruism and SDO have opposite effects on the same variable. From this, it could be extrapolated that SDO will have a negative relationship with altruism also. Since right-wing authoritarianism seems to promote a very selfish view of the world, the idea that it 'opposes' altruism, which encourages a selfless view is an easy link to make.

Sidanius et al. (2013) found data that supports this view in a cross-lagged study. Using a sample of Belgian students and New Zealand adults, they measured them on trait empathy and SDO at two different times. The results seemed to indicate that high levels of trait empathy at first measurement had a negative relation with SDO levels at second measurement and vice versa. Furthermore, trait empathy seemed to partially 
mediate the relationship between sex and SDO, and the relationship between sex and trait empathy was partially mediated by SDO.As feeling empathy is a common reason for altruistic actions (de Waal, 2008), the finding that SDO has a negative correlation with it over time serves as firm evidence for our hypothesis. However, we claim that charity adverts may make people less likely to donate towards the cause. If the reason for this is SDO, does this mean that SDO can fluctuate in someone?

\section{SDO as a plastic variable}

Huang and Liu (2005) conducted a study in Taiwan to test if SDO was a stable orientation towards hierarchies, or subject to external factors and priming. In the first study, using 1605 adults, they presented participants with questionnaires that would increase the salience of sexual differences or an arbitrary set-system (the demographic group they belonged to). The results suggested that men were higher in SDO only when primed on gender issues. In addition, participants who belonged to the Mingnan Taiwanese demographic group (the dominant group in the arbitrary set-system) scored higher on SDO compared to other groups in that set when primed with demographic group membership. A second study used 101 high school students visiting a museum during a field trip. In this study, they tested to see if priming different sets produced different levels of change in SDO. Participants had completed a pre-test questionnaire that primed them on demographic group membership (for example, by asking for opinions on intergroup relations between Taiwan and China). When visiting the museum, they completed a different questionnaire that primed them on race group membership. They found that priming race produced a much greater change in SDO than priming demographics. From this, they concluded that SDO is not a personality trait, but rather an ideological orientation that is not wholly stable across different contexts and situations (a belief shared by Duckitt, Wagner, du Plessis, \& Birum, 2002). If this is the case, then it may be possible that the accidental manipulation of SDO via a commercial that makes differences in demographics highly salient may hinder a charity drive. That is what this study aims to investigate.

As SDO appears to be a variable that can be primed, it could have an impact on behaviours associated with membership of salient intergroup categories. Because it has been correlated with many antisocial traits (Levin, Federico, Sidanius, \& Rabinowitz, 2002) (Sidanius, et al., 2013), one of those behaviours could be behaviours commonly 
seen as altruistic. Such behaviour includes intent on donating to charity, which will be used in this study. If we assume that SDO influence a decision to donate to charity, then priming SDO should have an influence on that decision. Given past research, this should hypothetically decrease any intent on donating to charity. In addition, given that SDO involves desiring inequality between demographics, if the charitable cause is one aimed at helping a minority or subordinate group; this effect should be even greater. As such, the goal of this study is to test the effects of priming SDO on the intent to donate to a charity aimed at helping a minority.

Furthermore, as SDO and RWA typically correlate on factors (Altemeyer, 1996), it is possible that we will see a similar effect of RWA on charitable intent. As such, we expect to see that RWA shares SDO's negative correlation with charitable intent.

However, before we investigate these main hypotheses, we wish to further investigate the measures we plan to use in the experiment. This required a separate study, which we will report now.

\section{Study 1A}

A criticism commonly aimed at the measure of SDO created by Sidanius and Pratto (1999) is that the wording of the definition has changed over time, resulting in the conflating of two sub-constructs of SDO (Rubin \& Hewstone, 2004). In the beginning, SDO was defined as "the degree to which individuals desire social dominance and superiority for themselves and their primordial groups over other groups." However, over time, its definition began to change, and now there are three distinct constructs all claiming to be Social Dominance Orientation. The definition from Pratto, Sidanius, Stallworth, and Malle, (1994) tried to expand the definition, but created two sub-constructs in doing so, holding that Social Dominance Orientation is about desiring ingroup dominance over outgroups, and also the desire for hierarchical relationships between groups in society. As such, SDO was split into two needs. The first is the need for ingroup dominance, which is called specific SDO while the second is the need for general intergroup hierarchies, which is called general SDO (Jost, Banaji, $\&$ Nosek, 2004). However, one of the consequences of splitting SDO is that the relation between SDO and ingroup status becomes difficult to deal with. Consider someone in a minority group with high SDO. With this split, they would have a conflict between the 
desire to see their group as dominant, caused by specific SDO, and desire to promote existing uneven intergroup hierarchies that they are low on, caused by general SDO. The second definition comes from Sidanius, Levin, Federico, and Pratto (2001). This definition eliminated specific SDO and kept general SDO. Their definition stated that SDO was the desire for unequal relations between social groups, regardless of whether one's ingroup would be dominant or subordinate. Meanwhile, the $\mathrm{SDO}_{6}$, one of the more common measures of Social Dominance Orientation (Pratto, Sidanius, Stallworth, \& Malle, 1994), defines it as the desire for unequal relations between social groups, regardless of the position one's ingroup falls into. As such, some feel that modern SDO actually consists of three constructs, rather than one (Rubin \& Hewstone, 2004).

As an example of how much the definition of Social Dominance Orientation has changed, consider the first definition of Social Dominance Orientation. This theory was originally designed to explain why low-status groups would display ingroup favouritism (Sidanius \& Pratto, 1999). Reicher (2004) had criticized it for only predicting that this ingroup favouritism was static across all low-status groups. However, the most recent definition of Social Dominance Orientation (Sidanius et al., 2013) is better at giving a reason as to the absence of any ingroup favouritism in minority groups (Rubin \& Hewstone, 2004). Because they are all so clearly distinct, the issue becomes that the evidence for the first two theories may not generalize to the most current one, as it is possible that the old measures were measuring something other than SDO. As such, they should be treated as three separate constructs.

Evidence for the idea that SDO consists of multiple dimensions comes from Jost and Thompson (2000). They had similar criticisms to Rubin and Hewstone (2004), claiming that past definitions of SDO had implicit assumptions that it captured both group-based dominance and anti-egalitarianism. Taking the SDO measure developed by Pratto, Sidanius, Stallworth, and Malle (1994), they pointed out that half the questions seemed to be about group-based dominance, taking the form of statements concerning how aggression and control should be used to keep subordinate groups in line. Meanwhile, the other half were anti-egalitarianism in nature, reverse-coded questions measuring disagreement towards statements concerning how we should be moving towards a more equal society. As such, their study attempted to find out whether or not group dominance and anti-egalitarianism were distinct ideologies, as well as how this might differ between European Americans and African Americans. 
Jost and Thompson (2000) conducted four studies, two of which used the original measures from Pratto, Sidanius, Stallworth and Malle (1994) and two which used an altered version in which half the items were reworded to be statements concerning group dominance ideology, while the other half were reworded to be statements concerning anti-egalitarianism ideology. Their results suggested that the SDO measure by Pratto et al. (1994) did mix together group dominance and antiegalitarianism. They also found evidence that group dominance and anti-egalitarianism were more strongly intertwined in high status groups like European Americans, as to oppose egalitarian movements advances the interests of their ingroup, they were less so for lower status groups like African Americans, where group dominance could correlate positively with self-esteem and ingroup value, but anti-egalitarianism was typically correlated negatively with such things.

Ho et al. (2012) followed up on Jost and Thompson (2000) by proposing a twofactor model of SDO, consisting of dominance and egalitarianism. Dominance strongly reflects the earliest definitions of SDO, as a measure of how much someone supports hierarchies of groups with the groups at the top actively suppressing the groups on the bottom. They also hypothesized that this sub-dimension would be correlated with support for negative intergroup attitudes, support for unequal allocation of resources between groups and an increased perception of competition between groups (Sidanius and Pratto, 1993). Egalitarianism, on the other hand, is a measure of how much someone opposes group equality. It differs from dominance, which is the support for active suppression of minority groups, by being a measure of how much someone supports exclusivity. Those who would score highly in egalitarianism do not necessarily want to oppress minority groups or support overt intergroup aggression. Rather, they would agree with legitimizing myths that support their position and social hierarchy as a whole. It would also correlate negatively with support for social policies that would threaten hierarchy by redistributing resources (Ho et al. 2012).

Ho et al. (2012)'s hypotheses were that a factor analysis of the $\mathrm{SDO}_{6}$ scale with multiple samples would all reveal these two sub-dimensions, which would be strongly correlated. They also hypothesised that different social settings would show moderating effects on these sub-dimensions, with dominance becoming more potent in settings where the social hierarchy is being actively contested. Egalitarianism on the other hand will become more potent in settings in which the social hierarchy is relatively stable. 
Using six samples from the United States and two from the Middle East, they had participants fill in the $\mathrm{SDO}_{6}$ scale, as well as measures of intergroup attitudes (with the exception of one sample that was drawn from a 1996 Los Angeles survey). Using confirmatory factor analysis, they found evidence that a two-factor model fit the data much more strongly than a one-factor model, with the measures of dominance and egalitarianism showing good reliability in all samples. In addition, tests for correlation between dominance and egalitarianism gave support for the idea that the two are highly correlated in all samples. Also, dominance did seem to correlate more strongly with the traits they thought it would correlate with in settings where the social hierarchy is being overtly challenged. In addition egalitarianism correlated more strongly with the traits they hypothesised it would in settings where the social hierarchy is relatively stable.

These results are a firm indication that SDO is more than a single dimension. However, it does use a version of Sidanius' SDO measure that is now outdated, thanks to Sidanius et al. (2013). In addition, there is the possibility that, while a two-factor model might fit the data better than a one-factor model, a model with more than two factors may be an even better fit than this two-factor model.

In an attempt to correct this, Ho et al. (In print) have developed a new measure of SDO that rectifies this by measuring four constructs that combine to form SDO. Looking at past research, they drew on evidence that Social Dominance Orientation is not unidimensional. Rather, it is multidimensional. In their study, Ho et al. (2012) reports evidence for the dimension of domination, the desire for the creation and maintenance of group-based hierarchies. In addition, they found evidence for antiegalitarianism, which is the desire for inequality between groups, but not domination in particular. Drawing on this research, Sidanius created a scale assessing Dominance and egalitarianism using both pro- and con-trait items.

These subcategories are positive-egalitarianism, negative-egalitarianism, positive-dominance and negative-dominance. The positive items measure how much an individual supports the traits and policies associated with each, and negative items measure how much they oppose them. The resulting scale uses four pro-trait and four con-trait items for both dominance and anti-egalitarianism. Their results indicated that a four-factor model best fit the data they got from five different sources, making the $\mathrm{SDO}_{7}$ scale an improvement over the previous scales. 
In addition, Pratto et al. (2012) also published a new measure to answer these criticisms. They went on a different tack however, by creating counter-dominance orientation (CDO). CDO is conceptualized as the opposite of SDO. It is the endorsement of ideologies such as multiculturalism and human rights shared by everyone. In the past, it was operationalized as scoring low on SDO scales. They claim that it exists as a response to oppressive social hierarchies, and caused by a need for social inclusion and a sense of belonging. The CDO scale consists of six items, all of which could be reverse-coded items on an SDO scale. Exploratory factor analysis conducted by Morselli et al. (2012) extracted two factors with three items each. However, these scales are still relatively untested, with the initial papers presenting them as of yet unpublished.

As the $\mathrm{SDO}_{7}$ is relatively new, and has yet to be tested in a non-US sample, before using them in our study, we set out to verify the psychometric structure of the scale. We hypothesize that we will find evidence that SDO is a multi-factor variable. Specifically, that it will be made up of four factors, based on past findings such as Ho et al. (In print) and Ho et al. (2012). Though this seems obvious, it is important to test and make sure that measures and variables developed in one culture work the same way in another (King, Murray, Salomon, \& Tandon, 2004). Furthermore, we hypothesize that a two-factor model will best fit CDO, based on the findings of Morselli et al. (2012).

\section{Method}

\section{Participants}

670 participants partook in the mass testing, all drawn from first year psychology students at Victoria University of Wellington. Of these participants, 495 were woman. 530 were Pākehā, while 37 were Māori. The remainder were classified as 'Other.' The youngest participant was 17, the oldest 53. The average age was 19.29.

\section{Apparatus/materials}

In this study, we used the $\mathrm{SDO}_{7}$ scale developed by Ho et al. (In print) to measure participant's levels of SDO. The scale consists of sixteen items, broken into four subsections. SDO-D measures dominance, the degree to which participants believed that force should be used to maintain social hierarchies. An example item from this section is "It's probably a good thing that certain groups are at the top and other 
groups are at the bottom." SDO-E measures anti-egalitarianism, how much people endorse policies that maintain unequal social hierarchies. An example item is "It is unjust to try to make groups equal." The other two subsections are reverse-coded items of these two earlier subsections, SDO-DR and SDO-ER. Past research, such as Jylhä \& Akrami (2015) have used the study and found it to be reliable $(\alpha=0.85)$.

We also used the CDO scale developed by Pratto et al. (2012) to gather data from participants. This scale consists of six items split into two groups. The first is $\mathrm{CDO}$, and measures how much participants endorse social harmony and reduced gaps between hierarchies. For example, one item from this scale is "There's nothing more important than confronting oppression by dominant groups." The second subscale is CDO-R, consisting of reverse-coded items. For example, "Sometimes it's right for a subordinated group to compromise with a dominant group." The combined $\mathrm{SDO}_{7}$ and CDO scales can be viewed under Appendix A. In order to analyze the data, we used IBM SPSS for reliability analysis and IBM SPSS Amos for confirmatory factor analysis (CFA).

\section{Procedure}

First-year psychology students completed the $\mathrm{SDO}_{7}$ as part of a set of 'mass testing' online measures and tasks, during the first week of the laboratory course, and at a time of their own convenience. After removing participants who failed to answer one or more items, we were left with a sample of 670. Following this, the data gathered for each measure underwent CFA using IBM SPSS Amos. CFA is a method of testing a hypothetical model for how a factor and sub-factors interact. This allowed us to input the theoretical models of how many factors each scale could be measuring. This is a stronger method of factor analysis than exploratory factor analysis (EFA), as EFA is a data-driven analysis that attempts to maximise how much variance is explained without incorporating any hypotheses the experimenter has. CFA, on the other hand, requires the experimenter to input the model to be tested against the data, including how each item might load onto each factor, and gives an indication of how well the data fits.

By comparing the results of different hypothetical structures, we can test to see whether or not SDO and CDO better fit their hypothesized models. In addition, we will be testing for the reliabilities of these models, so as to see if either of them seems more reliable and thus, better suited for future studies than the other. 


\section{Results}

A confirmatory factor analysis was conducted on Sidanius et al's $\mathrm{SDO}_{7}$ scale, and Pratto et al's CDO scale. To conduct this analysis, the dataset was imported from IBM SPSS, and then hypothetical models were designed. The models consisted of the hypothesized latent variables, linked to each observed variable (question responses from the surveys) expected to be attributed to them.

For $\mathrm{SDO}_{7}$, a one-factor (with all variables loaded onto a single 'SDO' factor), two-factor (with half the variables loaded onto an SDO-dominance factor and the other half loaded onto an SDO-egalitarianism factor, with the two expected to correlate), and four-factor (with the variables quartered and loaded onto 'positive SDO-dominance,' 'negative SDO-dominance, ' 'positive SDO-egalitarianism,' and 'negative SDOegalitarianism') model was tested. For CDO, a one-factor (with all variables loaded onto a 'CDO' factor) and two-factor (with half the items loaded on to CDO-positive and the other half loaded on to CDO-negative) model was tested.

Results of the confirmatory factor analysis, as well as the reliability test are presented in tables 1 and 2 . The data indicates that, out of the one, two, and four-factor models for SDO, the four-factor model has the best fit. Meanwhile, between the one and two-factor model for CDO, the two-factor model has the best fit. Furthermore, the most fitting models are presented in Figures 1 and 2.

In addition, to test for the possibility that SDO and CDO might be conflated with each other, another model was tested. In this model, SDO-D and -E were correlated with CDO-R and SDO-DR and -ER were correlated with CDO. This model was insignificant $\left(\chi^{2} / d f=20.69, \mathrm{RMSEA}=0.17, \mathrm{CFI}=1.00\right)$ and is presented in Figure 3.

\section{Discussion}

The results of the CFA seem to indicate that the four-factor model of SDO is the best fit for the data gathered. The chi-squared test falls quite close to zero, which suggests there is a small difference between expected and observed covariance matrices. The RMSEA falls below .06, indicating an acceptable goodness-of-fit in spite of the large sample. The CFI is above .90, which indicates relatively little discrepancy between the data and the hypothesized model. Also, the scale reliability falls above .80 , which implies a good 
Table 1. Fit statistics for confirmatory factor analysis of one, two and four-factor models of SDO, along with the reliability of the measure.

\begin{tabular}{ccccccc}
\hline Model & $\chi^{2}$ & $D f$ & $\chi^{2} / d f$ & RMSEA & CFI & $\begin{array}{c}\text { Reliability } \\
(\alpha)\end{array}$ \\
\hline $\begin{array}{r}\text { One- } \\
\text { factor }\end{array}$ & 739.28 & 104 & 7.11 & .094 & .83 & \\
$\begin{array}{l}\text { Two- } \\
\text { factor }\end{array}$ & 698.61 & 103 & 6.78 & .092 & .84 & .87 \\
$\begin{array}{l}\text { Four- } \\
\text { factor }\end{array}$ & 284.61 & 98 & 2.90 & .053 & .95 & \\
\hline
\end{tabular}

Table 2. Fit statistics for confirmatory factor analysis of one and two-factor models for counter-dominance orientation (CDO) measure, as well as the reliability of the measure.

\begin{tabular}{ccccccc}
\hline Model & $\chi^{2}$ & $d f$ & $\chi^{2} / d f$ & RMSEA & CFI & $\begin{array}{c}\text { Reliability } \\
(\alpha)\end{array}$ \\
\hline $\begin{array}{r}\text { One- } \\
\text { factor }\end{array}$ & 117.71 & 9 & 13.08 & .13 & .79 & \\
$\begin{array}{r}\text { Two- } \\
\text { factor }\end{array}$ & 19.51 & 8 & 2.44 & .044 & .98 & \\
\hline
\end{tabular}

internal consistency between the items. As such, $\mathrm{SDO}_{7}$ appears to be a reliable fourfactor model, suitable for future social dominance studies.

But, there is the possibility that the high reliability observed is an artefact created by the large number of scale items. As the $\mathrm{SDO}_{7}$ scale has sixteen items, the high reliability we observe could be inflated due to that large number. Alphas have a curvilinear relationship with the number of items, one that begins to level off as number of items approaches 19 (Cortina, 1993). As $\mathrm{SDO}_{7}$ has 16 items, it has begun to approach this levelling off, so this becomes a very real concern.

Meanwhile, the two-factor model of CDO seems to be the best fit. Like the fourfactor SDO model, it meets the requirements of the absolute fit indices that were looked at. However, its reliability falls into the 'questionable' range of Cronbach's alpha (Gliem 
Figure 1. Four-factor $\mathrm{SDO}_{7}$ model.

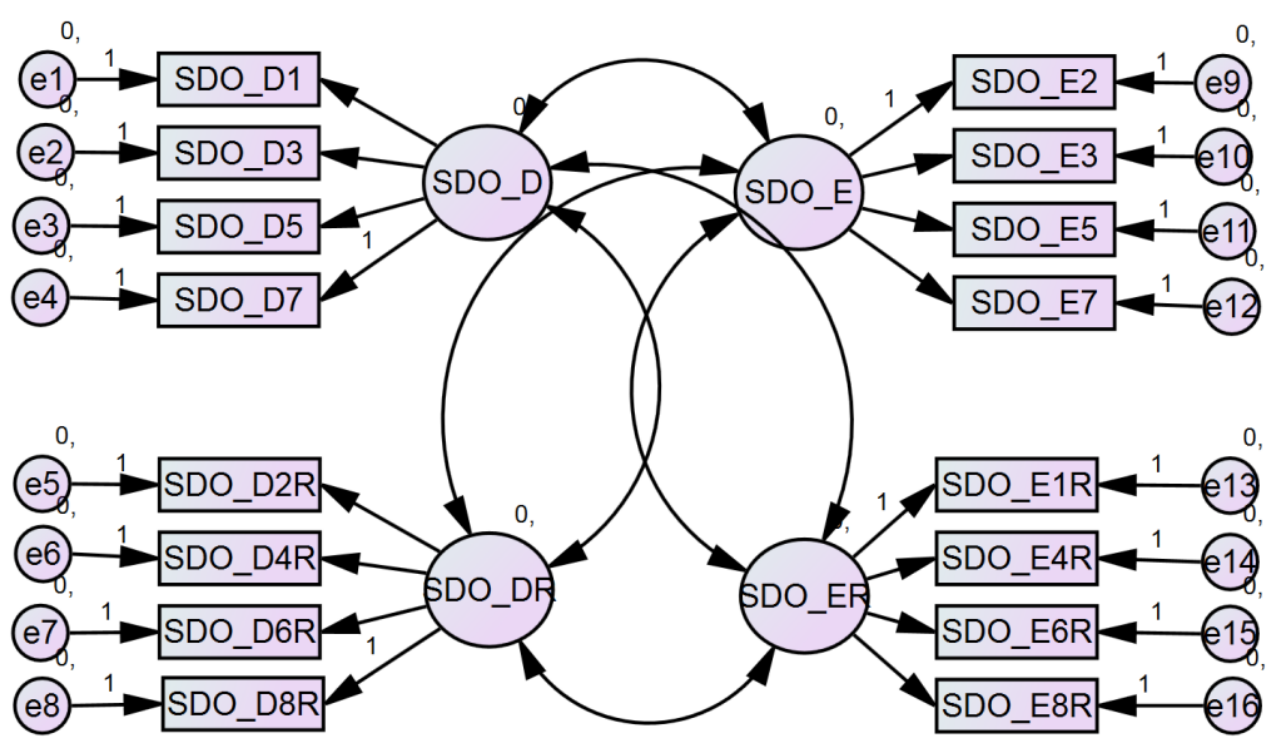

Figure 2. Two-factor CDO model.

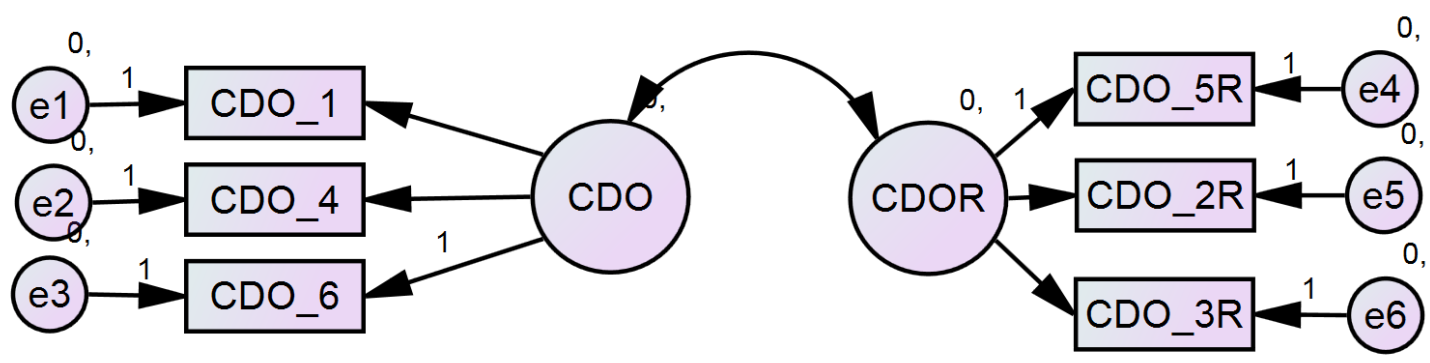

Figure 3. SDO-CDO model

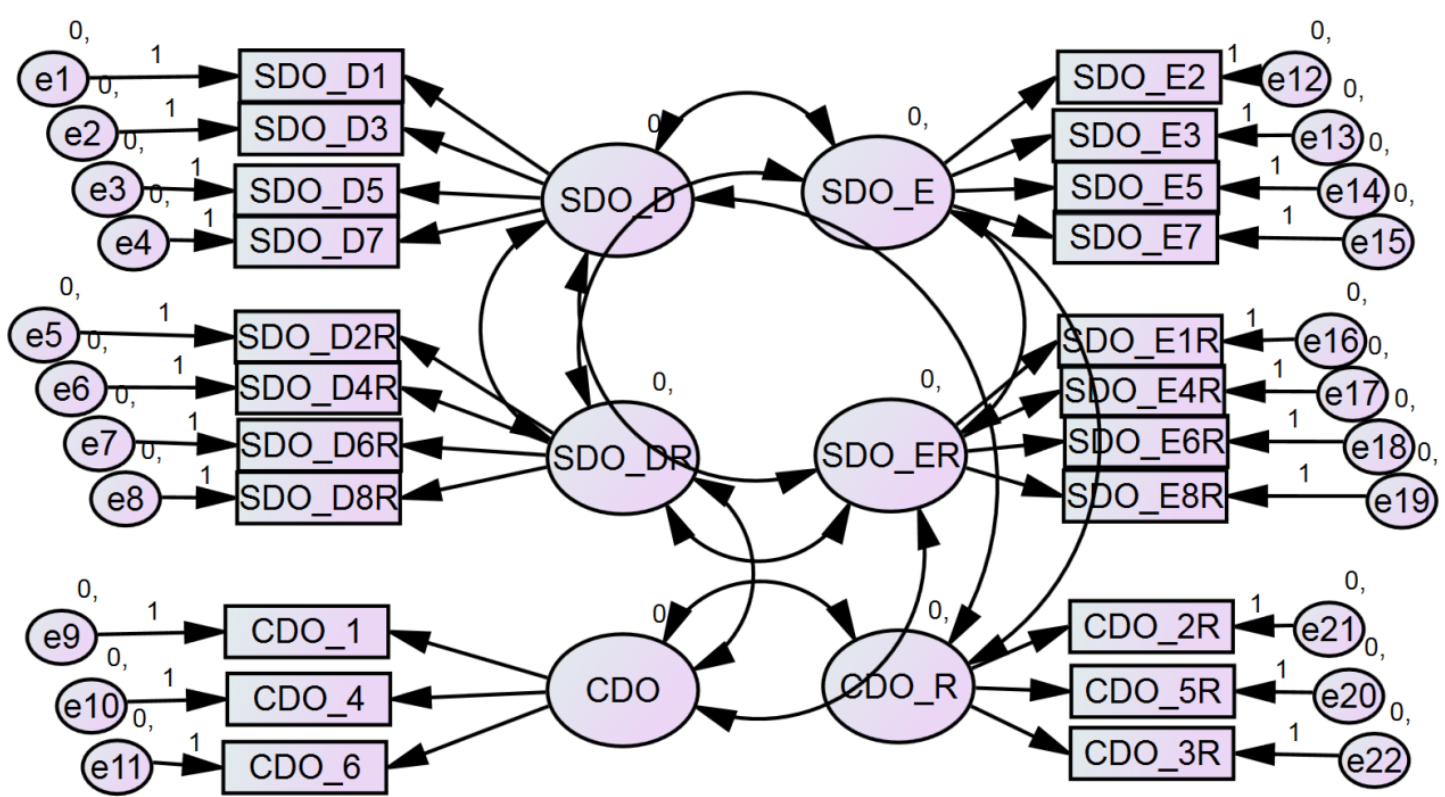


\& Gliem, 2003), which suggests it may not be the best scale to use in SDO studies. In addition, it scored much lower than $\mathrm{SDO}_{7}$, at times not even falling within the recommended level of reliability (Gatignon, 2010; Hu \& Bentler, 1999).

As a result of these findings, one can conclude that the CDO scale, as it stands, may need improvement before it can be more widely utilized in research. One recommendation we would make would be to expand the number of questions it asks. As it stands, six questions with three for each sub-factor may not be enough to properly measure a participant's CDO. Just as a high number of items can inflate the alpha of a measure, a low number of items can deflate the alpha (Cortina, 1993). At the bare minimum, we would recommend twelve questions, with six for each sub-factor. However, our attempts to build a model that might show how CDO could be conflated with SDO were met with frustration. Both models used showed poor model fit. These findings could be construed as evidence for the existence and validity of CDO as a variable separate from SDO. Were the two actually strongly conflated, we might observe a well-fit model in which the two are correlated. As we have failed to do so, this could mean that the two truly are separate.

Some may argue that, with all these issues CDO currently suffers from, it may be simpler to continue utilizing SDO for the time being. We, however, feel that CDO represents something that should be encouraged, as it represents a way that positive psychology can enter the field of social psychology. Positive psychology is the study of human thinking and behaviour with the goal of identifying what is good, just and virtuous in the human condition. It holds that modern psychology has taken a dim view on what a happy, thriving person's psyche is like, in favour of learning more about damaged psyches, or those that tend to display negative affect (Sheldon \& King, 2001).

SDO is easy to categorize as a negative factor, as it seems to correlate negatively with things like welfare programmes (Sibley \& Duckitt, 2010), and trait empathy (Sidanius et al., 2013). However, when your only means of measuring a variable is by defining it in negative terms that can lead to an unfortunate implication, in which people who score low in such a variable are still counted as possessing some of such a variable. In addition, those who score high in such a variable may begin to feel uncomfortable with the idea that they possess quantities of a trait that would be frowned upon by others. But, if we refined and began using CDO more, this would at least solve the first 
issue. People who score low in SDO could then be counted as scoring high in CDO, which is a much more positive way to frame the situation. Because CDO encapsulates qualities such as opposition to oppressive regimes and supporting those who are a victim of them (Pratto et al., 2012), it is a far more virtue-oriented alternative to SDO, and thus, more suited to a positive psychology.

In addition, during this study, there was an ongoing issue of participants who failed to answer all the items on the measures used. One possible reason for this could be that they were affronted by the question topic. Consider this item from the dominance subset of $\mathrm{SDO}_{7}$, "It's probably a good thing that certain groups are at the top and other groups are at the bottom." People who are filling in the measure may be offended at the statement, feeling that the measure is asking how racist/classist they are and thus, refusing to give an answer. Not only would this reflect poorly on the measure, as the items were not sufficiently neutral enough to reduce the risk of response bias (Nederhof, 1985), it means that analyses with the measure will be stymied by incomplete answers. This would require omitting data and reducing the sample or filling them in with an average, which could result in inaccurate results (Downey \& King, 1998). Using a CDO measure might be more suitable, as the items tend to have a more positive leaning, reducing the chance that participants refuse to answer them.

\section{Study 1B}

Now that we have ascertained that the $\mathrm{SDO}_{7}$ scale is reliable and fits the proposed model, we now move on to the study that will form the main focus of this paper. As mentioned previously, Huang and Liu (2005) found evidence that SDO can be primed, leading them to score higher in measures of it. This finding can be quite useful to SDO researchers, as it partially eliminates the need of a large sample and an arbitrary division between participants of high and low SDO. Now, they can simply prime a group of participants on SDO and compare their results with a group who was not primed. However, this raises the possibility that stimuli that people encounter in everyday life may unintentionally prime them on SDO. This could potentially occur even in areas where priming people on SDO would be disadvantageous. One area that this study is focussed on is charity advertisement.

Advertising consists of any form of communication aimed at getting people to undertake or continue with a certain action. Thus, charity advertising is a form of 
communication intended to get people to donate money to the charity in question. Many techniques exist to make such advertisements incentivise viewers to donate to the charity. Das, Kerkhof, and Kuiper (2008) carried out an investigation on what makes a charity advertisement effective. Using a 2 × 2 × 2 study to create eight different adverts, they tested the effects of three types of information on intent to donate to a charitable cause. The first was whether or not participants got to see information on how many have donated to the campaign already. The second was whether anecdotal information or statistical information was more effective. The third was whether or not focusing on the positive outcomes of donating was more effective compared to focusing on the negative outcomes of not donating.

Results suggested that participants liked seeing how close the fundraising campaign was to meeting its goal, and they also seemed to prefer anecdotal information over statistical information. However, participants viewed the charity's work as more relevant when anecdotal evidence was presented positively and also when statistical information was presented negatively. However, the single factor that seemed to influence willingness to donate the most was the presence of fundraising goals.

Chang and Lee (2011) looked at how the different sexes respond to altruistic and egotistic charitable appeals, as well as how self-referencing can influence the processing of these appeals. In this case, self-referencing refers to the processes people use to understand incoming information that is relevant to them by comparing it to alreadyheld information that they have stored in memory. Participants were asked to appraise an advertisement of a charity in the form of a poster. Posters for the egotistic message involved appeals to capability, the protection of loved ones and logic values. Posters for the altruistic message involved appeals to love, equality and social responsibility. Selfreferencing was manipulated by having posters for elderly welfare as the high selfreferencing condition (as everyone will get old one day) and disability welfare as low self-referencing.

The results fell in line with their hypotheses, indicating that men being more influenced by egoistic advertisements when self-referencing was high, while women were more influenced by altruistic advertisements when self-referencing was high. This study is evidence for the idea that advertisements can have different effects on people based on sex. However, sexes are not an arbitrary-set difference, and so not exactly the 
same as what the current study is looking at. What evidence is there that advertisements can have a different effect on people based on what arbitrary-set ingroups they fall into?

A study that investigates this is Nelson, Brunel, Supphellen, and Manchanda (2006). They looked at whether or not advertising would influence a viewer's perception on moral obligations to help others, using samples drawn from nations that differed on average levels of masculinity. In this case, masculinity refers to the level of assertive, egocentric interests, and is contrasted with femininity, which is the level of supportive, nurturing interests. The four samples were from the United States and Canada as the high masculinity samples, and Denmark and Norway as the low masculinity samples. Participants responded to egocentric or altruistic charitable demands. Results indicated that men from masculine nations responded more favourably towards egocentric appeals, with women responding more positively towards altruistic appeals. However, in less masculine nations, the reverse pattern was observed. In addition, participants from masculine cultures reported more moral obligation to help others after exposure to an altruistic advertisement when compared to exposure to an egocentric advertisement. Participants from a less masculine culture, on the other hand, showed less moral obligation to help others when exposed to the altruistic advertisement. In addition, they were more likely to believe that the government had an obligation to help others than they did. These findings support the idea that culture of origin can also influence how people perceive and respond to charitable advertisements.

In addition, Bennett (1997) investigated how presenting the unpleasant reality of what the charity aims to help (in this case, facial disfigurement) will influence empathy and emotionality. Participants were presented a child with a disfigured face on their own, a disfigured child alongside an unattractive female, or the child alongside an attractive female. Alongside these visual stimuli was a written appeal for a charity for helping those with facial disfigurement. Results indicated that, compared to a control group who only saw the female with no facially disfigured child, participants showed the most favourable responses to an attractive female, with the disfigured child given less prominence. Even just the attractive woman inspired a more empathetic response from participants than the disfigured child on their own. 
These results could imply that, contrary to what seems to be common sense, simply presenting the reality of the situation that charities wish to help change does not inspire the most charitable responses from participants. In addition, the findings from Das et al. depend on the idea that people from different groups will respond to charity advertisements in the same way. But what if this is not the case? Is it possible that people from different groups may respond differently to the same advertisement? These two ideas, that people might not respond to charitable advertisements in expected ways, and that different groups may show different responses to the same advertisements, are the core of this study.

\section{Method}

\section{Participants}

First-year psychology students were invited to participate in the study as part of a mandatory research experience course component. Students can choose to complete this requirement either through participation in research, or reading and summarising research articles. The number of participants, after removing those who were not also in Study 1A, was 139. 45 participants were male, while 94 were female. 97 participants were NZ European/Pākehā, while 12 were Māori, and 31 were categorized as 'other.' $87.2 \%$ of participants fell in the age range of 18-20, with the youngest being 17 and the oldest being 53 .

\section{Apparatus/materials}

Scales used in this study consisted of the $\mathrm{SDO}_{7}$ scale (Ho, et al., in print); the CDO scale (Pratto, et al., 2012) and the RWA scale (Altemeyer, 1996). The stimulus used to prime participants on social dominance consisted of an advert for a service aimed at helping students at Victoria University of Wellington to excel. Two versions were used. In both versions a generic description of University support services was provided:

"University study is now an option for more New Zealanders than ever before. This also means that people coming to university come with very different experiences, skills, and understandings of what it means to be a student. Additionally, study is just one part of people's lives, and sometimes people need a little support to help them achieve the best results that they can. 
For these reasons, universities typically offer a range of services, organisations, and opportunities to work alongside students. Typically, this might involve assigning a mentor for new students, chances to meet with people to work through what is expected in assignments, advice on foundation skills necessary to succeed at university, or a place to study and putting students in contact with others like them."

The two versions differed in terms of who the service's target audience was (with examples of services for those groups) and picture. The first version referenced supports for Māori and Pacifica, and gave as an example the Faculty of Science. This advert also included a picture that incorporated typically Māori and Pacifica images. In this picture, a group of people of Māori and Pacifica descent, standing in front of a marae (Māori meeting house). The specific advert can be found in Appendix C. The other was an advert for general student support, and gave as an example Student Learning Support, an organization to teach all students good study habits. The picture in this advert was more racially neutral, depicting a mix of students from a mix of racial groups studying together. The specific advert can be found in Appendix D.

The adverts used the same body of text, deliberately worded so as to refer to both agencies without any falsehoods, with the only difference being the names used. After participants looked at the advert, they were asked to critically evaluate the advert and the agencies they were for. They were asked how effective and important they felt such services were, and asked to comment on how effective they felt the advert would be in attracting people to use it.

Their answers were measured on a seven-point Likert scale, with one being 'not at all important/effective' and seven being 'very important/effective.' They were also asked to put down what percentage of students they believed used that service. These questions can be found under Appendix E. Also used was a 'tragedy of the commons' question, similar to the one used in Marwell and Ames (1979). This question asked:

"Imagine a hypothetical scenario in which, in addition to IPRP credit, you also received $\$ 5$ for your participation in this study.

Imagine that you could either keep the $\$ 5$, or donate a portion of it into a public pool of money. Your fellow participants would have the same opportunity. Any money in the public pool would be doubled, and then distributed evenly across the participants as 
well as [the name of the organizations listed in the advert they viewed]. You and the other participants would not be allowed to discuss it in any way until after the money has been handed out.

In this hypothetical scenario, how much money would you put into the public pool?"

Both versions of the above question can be found under Appendix F (the experimental version) and Appendix G (the control version).

\section{Procedure}

Participants, upon giving consent to be a part of the online study, were randomly assigned to one of the two conditions that split participants based on the advert they saw. They were shown the advert, and then asked to critically evaluate it. Following this, they would fill in the RWA scale and the $\mathrm{SDO}_{7}$ and CDO scales, whose items were mixed together. Then, they were asked to supply an answer to the public goods game, while the picture they saw with the advert was presented again, so as to remind/re-prime the participant.

This marked the end of the study, and participants were debriefed via text. As we had enough participants common to the data used in Study 1A and this study to create a sample size large enough for this study, the data from both studies was merged, and participants who only participated in one of the two studies were eliminated (we had measured participants in study 1A on RWA, but the data was not used for that study). In doing this, we had pre-test and a post-test data on these participants, allowing for tests to examine the effects of the SDO priming. Although the loss of so many participants and their data may weaken our results, we felt that using pre-test data would allow us to more conclusively prove whether or not our experimental group was primed on SDO. Because we had measured their SDO earlier, we would be more able to gauge any changes in their SDO in study 1B as we had pre-test data on their SDO.

\section{Results}

Means and standard deviations of the Pākehā students on all outcome variables were calculated, and are presented in Table 3. Means and standard deviations for both experimental and control conditions on all outcome variables were also calculated, and are presented in Table 4. 
Tests of Social Dominance on Charitable Intent Towards Minorities

Table 3. Overall means and standard deviations of all outcome variables.

\begin{tabular}{ccc}
\hline & Mean & SD \\
\hline Student service effectiveness & 6.55 & 1.87 \\
Student service importance & 8.12 & 1.85 \\
Advert effectiveness & 5.35 & 1.77 \\
Student service usage (\%) & 44.91 & 19.01 \\
Hypothetical donation & 4.32 & 2.81 \\
\hline
\end{tabular}

Table 4. Means and standard deviations split by condition on outcome variables

\begin{tabular}{ccccc}
\hline & \multicolumn{3}{c}{ Condition } \\
& \multicolumn{2}{c}{ Experimental } & \multicolumn{2}{c}{ Control } \\
& Mean & SD & Mean & SD \\
\hline Student service effectiveness & 6.30 & 1.79 & 6.74 & 1.92 \\
Student service importance & 8.03 & 1.88 & 8.20 & 1.83 \\
Advert effectiveness & & & & \\
Student service usage $(\%)$ & 5.48 & 1.58 & 5.26 & 1.91 \\
Hypothetical donation & 44.18 & 18.49 & 45.51 & 19.52 \\
& & & & \\
& 4.25 & 3.29 & 4.37 & 2.40 \\
\hline
\end{tabular}

Afterwards, a MANOVA was conducted to test for differences between the two conditions on these outcome variables. Results suggest that there is no difference between the two conditions in general $\left(F(5,122)=.49, p=.78\right.$, Wilks $\Lambda=.98, \eta^{2}=$ .020). Specifically, condition had no significant influence on perceived effectiveness 
$\left(F(1,126)=1.31, p=.26, \eta^{2}=.010\right)$, importance $\left(F(1,126)=.61, p=.44, \eta^{2}=.005\right)$, effectiveness $\left(F(1,126)=.15, p=.70, \eta^{2}=.001\right)$, percentage of students who use them $\left(F(1,126)=.75, p=.39, \eta^{2}=.006\right)$, and hypothetical donation amount $(F(1,126)=$ $\left..049, p=.82, \eta^{2}<.001\right)$.

Another MANOVA was then conducted, using participant demographic variables to test for differences across sex and ethnicity. For this analysis, the ethnicity filter was removed, and all participants were used. The results indicated a significant difference between the sexes on the observed results $(F(5,173)=2.31, p=.046$, Wilks $\left.\Lambda=.94, \eta^{2}=.063\right)$, but no difference between ethnicities $(F(10,346)=1.51, p=.14$, Wilks $\left.\Lambda=.92, \eta^{2}=.042\right)$. There was also no interaction between the two demographic variables. $\left(F(10,346)=1.02, p=.43\right.$, Wilks $\left.\Lambda=.94, \eta^{2}=.029\right)$. However, upon looking at each independent variable, the only significant difference between the sexes was on the percentage of students they believed used student services $(F(1,177)=8.23$, $\left.p=.005, \eta^{2}=.044\right)$. Specifically, it appears that women $($ Mean $=49.18, \mathrm{SD}=19.46)$ believed that a greater percentage of students used such services than men (Mean $=$ $39.17, \mathrm{SD}=17.84)$.

It is worth noting that there was a significant difference between ethnicities on perceived effectiveness of student services $\left(F(2,177)=3.05, p=.050, \eta^{2}=.033\right)$, with Māori (Mean = 7.93, SD = 1.82) and 'Other' (Mean =7.19, SD = 1.78) participants rating them more effective than Pākehā $($ Mean $=6.53, \mathrm{SD}=1.84)$. Also, there was a significant difference on perceived importance $\left(F(2,177)=3.48, p=.033, \eta^{2}=.038\right)$ with Māori (Mean $=9.50, \mathrm{SD}=0.85)$ viewing student services as more important than participants in the 'Other' $($ Mean $=8.67, \mathrm{SD}=1.49)$ category, with Pākehā $(\mathrm{Mean}=$ $8.11, \mathrm{SD}=1.89)$ viewing them as the least important.

Means, standard deviations, and reliabilities were calculated for all scales used, both for pre-and post-test, both as a whole and by condition. 99 valid cases of Pākehā participants were used to calculate these for the pre-test (participants who had completed all measures at both pre- and post-test), and the results are presented in Table 5. For the post-test, means and standard deviations were calculated based on condition, with reliabilities calculated using the whole sample.

Then, a paired samples t-test was conducted on the results of each scale at pretest and post-test, to test for significant differences in the results observed over time. A 
Table 5. Means, SDs and reliabilities for all scales used in the study at pre- and posttest, with post-test means and SDs split by condition.

Pre-test

Post-test

Condition

Experimental Control

\begin{tabular}{ccccccccc} 
& Mean & SD & Reliability & Mean & SD & Mean & SD & Reliability \\
\hline $\mathrm{SDO}_{7}$ & 2.87 & 1.01 & 0.91 & 2.80 & 1.00 & 2.59 & 0.95 & 0.92 \\
$\mathrm{SDO}_{7}$-D & 3.04 & 1.08 & 0.82 & 2.87 & 1.00 & 2.78 & 1.00 & 0.83 \\
$\mathrm{SDO}_{7}$-E & 2.71 & 1.08 & 0.86 & 2.73 & 1.10 & 2.41 & 0.99 & 0.87 \\
$\mathrm{RWA}$ & 2.62 & 0.88 & 0.93 & 2.61 & 0.91 & 2.49 & 0.93 & 0.95 \\
$\mathrm{CDO}$ & 4.18 & 0.82 & 0.62 & 4.11 & 0.86 & 4.39 & 0.92 & 0.62 \\
\hline
\end{tabular}

Note: Post-test reliabilities were calculated using data from both conditions.

significant difference was observed with $\mathrm{SDO}_{7}(t(93)=2.08, p=0.04)$ and $\mathrm{SDO}_{7}-\mathrm{D}$ $(t(93)=2.36, p=0.02)$. However, there was no significant difference between RWA $(t(93)=1.79, p=0.078), \mathrm{SDO}_{7}-\mathrm{E}(t(93)=1.41, p=0.16)$ or $\mathrm{CDO}(t(93)=-1.54, p=$ 0.13 ) between the two time periods.

An independent-samples t-test was conducted to test for differences between the two conditions on measured variables. No significant difference between the conditions was found for $\mathrm{SDO}_{7}(t(139)=1.30, p=.20), \mathrm{SDO}_{7}-\mathrm{D}(t(139)=0.55, p=.59), \mathrm{SDO}_{7}-\mathrm{E}$ $(t(139)=1.81, p=.071)$, RWA $(t(139)=0.84, p=.40)$, or $\operatorname{CDO}(t(139)=-1.89, p=$ $.061)$.

Several repeated measures ANOVAs were then conducted to conclusively test for any effect of time as a within-subjects effect and condition as a between-subjects effect on all measured variables. Using Greenhouse-Geisser corrections, so as to account for the issue of increasing variability over time, we did not find a significant difference between pre-test and post-test measures for $\mathrm{SDO}_{7}(F(1,92)=3.48, p=.065)$, nor any differences as a result of condition $(F(1,92)=0.29, p=.59)$, and there was not any significant differences as a result of interactions between time and condition $(F(1$, $92)=1.84, p=.18)$. We did observe a significant effect of time on $\mathrm{SDO}_{7}-\mathrm{D}(F(1,92)=$ $4.60, p=.035$ ) with $\mathrm{SDO}_{7}$-D being greater at the pre-test than the post-test, but condition did not have a significant effect $(F(1 ., 92)=1.23, p=0.27)$ and there appeared to be no interaction $(F(1,92)=1.87, p=0.18)$. 
Tests of Social Dominance on Charitable Intent Towards Minorities

Furthermore, $\mathrm{SDO}_{7}$-E was not significantly affected by time $(F(1,92)=1.52, p=$ $0.22)$, condition $(F(1,92)=0.002, p=.96)$, nor by an interaction of the two $(F(1,92)=$ $1.14, p=0.29)$. RWA was near-significantly affected by time $(F(1,92)=3.26, p=$ $0.074)$, but apparently unaffected by condition $(F(1,92)=0.013, p=0.91)$ with no significant interaction between time and condition $(F(1,92)=0.11, p=.074)$. Finally, CDO was not significantly influenced by time $(F(1,92)=1.75, p=.19$,$) , condition$ $(F(1,92)=.063, p=.80$,$) nor by an interaction of the two (F(1,92)=1.96, p=.17)$.

Correlations between all outcome variables were then calculated, and are presented in Table 6. Then, correlations between all psychological and outcome variables were calculated, and are reported in Table 7. It is important to note that all variables save $\mathrm{CDO}$ had a significant correlation with the hypothetical amount donated. This implies that, even if the manipulation did not have a significant effect, the variables it was intended to influence do seem to correlate with intent to donate in the hypothesized way.

Each of the outcomes variables was regressed onto $\mathrm{SDO}_{7}, \mathrm{RWA}$ and CDO. For effectiveness of student services, neither $\mathrm{SDO}_{7}(\beta=-0.072, t(137)=-0.061, p=.55)$, RWA $(\beta=-0.17, t(137)=-1.56, p=.12)$, nor $\operatorname{CDO}(\beta=0.10, t(137)=0.96, p=.34)$ predicted scores. However, together they predicted a significant amount of variance in effectiveness scores $\left(R^{2}=.088, F(3,137)=4.40, p=.005\right)$.

As for importance of student services, $\mathrm{SDO}_{7}(\beta=-0.29, t(136)=-2.55, p=0.012)$, and RWA $(\beta=-0.23, t(136)=-2.26, p=0.27)$ significantly predicted scores, but not CDO $(\beta=-0.12, t(136)=-1.11, p=0.27)$. Again, all three significantly predicted variance in scores $\left(R^{2}=0.16, F(3,136)=9.05, p<.001\right)$. Another regression was done while omitting CDO, and the amount of variance predicted went up $\left(R^{2}=0.16, F(2,137)=\right.$ 12.94, $p<.001)$. Regressing $\mathrm{SDO}_{7}(\beta=-0.080, t(137)=-0.65, p=0.52)$, RWA $(\beta=$ $1.33, t(137)=1.19, p=0.24)$ and $\operatorname{CDO}(\beta=0.13, t(137)=1.15, p=0.25)$ onto advertisement effectiveness revealed no significant predication of scores. Together, they failed to predict any significant variance in scores $\left(R^{2}=0.022, F(3,137)=1.03, p=\right.$ $0.38)$. 
Tests of Social Dominance on Charitable Intent Towards Minorities

Table 6. Correlations between all outcome variables.

\begin{tabular}{ccccc}
\hline & $\begin{array}{c}\text { Student service } \\
\text { importance }\end{array}$ & $\begin{array}{c}\text { Advert } \\
\text { effectiveness }\end{array}$ & $\begin{array}{c}\text { Student } \\
\text { service use } \\
(\%)\end{array}$ & $\begin{array}{c}\text { Hypothetical } \\
\text { donation }\end{array}$ \\
\hline $\begin{array}{c}\text { Student service } \\
\text { effectiveness } \\
\text { Student service } \\
\text { importance } \\
\text { Advert } \\
\text { effectiveness } \\
\text { Student service }\end{array}$ & $0.43^{* *}$ & $0.17^{*}$ & 0.16 & 0.13 \\
use $(\%)$ & - & $0.24^{* *}$ & 0.16 & $<0.01$ \\
\hline
\end{tabular}

Note: $*$ indicates correlation significance at $p<.05$. ** indicates correlation significance at $p<.01$

Table 7. Correlations between all psychological variables and outcome variables.

\begin{tabular}{cccccc}
\hline & $\begin{array}{c}\text { Student } \\
\text { service } \\
\text { effectiveness }\end{array}$ & $\begin{array}{c}\text { Student } \\
\text { service }\end{array}$ & $\begin{array}{c}\text { Advert } \\
\text { importance }\end{array}$ & $\begin{array}{c}\text { Student } \\
\text { service }\end{array}$ & $\begin{array}{c}\text { Hypothetical } \\
\text { effectiveness } \\
\text { use } \\
\end{array}$ \\
& $-0.25^{* *}$ & $-0.36^{* *}$ & -0.08 & 0.03 & $-0.27^{* *}$ \\
\hline $\mathrm{SDO}_{7}$ & $-0.29^{* *}$ & $0.35^{* *}$ & -0.03 & 0.03 & $-0.28^{* *}$ \\
$\mathrm{SDO}_{7}-\mathrm{D}$ & $-0.19^{*}$ & $-0.34^{* *}$ & -0.11 & 0.04 & $-0.23^{* *}$ \\
$\mathrm{SDO}_{7}-\mathrm{E}$ & $-0.27^{* *}$ & $-0.36^{* *}$ & 0.01 & -0.06 & $-0.21^{*}$ \\
$\mathrm{RWA}^{* *}$ & $0.24^{* *}$ & $0.20^{*}$ & 0.11 & 0.03 & 0.17 \\
$\mathrm{CDO}$ & & & &
\end{tabular}

Note: * indicates correlation significance at $p<.05$. ** indicates correlation significance at $p<.01$

As for perceived percentage of students who use student services, $\operatorname{SDO}_{7}(\beta=$ $0.15, t(135)=1.23, p=0.22)$ failed to predict scores, same with RWA $(\beta=-0.12, t(135)$ $=-1.01, p=0.29)$ and $\operatorname{CDO}(\beta=-0.66, t(135)=0.58, p=057)$. Together, they failed to predict any variance in scores $\left(R^{2}=0.014, F(3,135)=0.66, p=0.58\right)$. 
Finally, regressing $\mathrm{SDO}_{7}$ onto the amount donated revealed a near-significant ability to predict the donated amount $(\beta=-0.24, t(127)=-1.90, p=0.06)$. However, RWA $(\beta=-0.063, t(127)=-0.56, p=0.58)$ and CDO $(\beta=-0.013, t(127)=-0.12, p=$ 0.91 ) had no significant effect. Together, the three were able to significantly account for variation in the donated amount $\left(R^{2}=0.074, F(3,127)=3.37, p=0.021\right)$.

\section{Discussion}

Our main hypothesis was not supported. Our experimental priming stimulus appeared to have no impact on SDO, and there was no difference between the control and experimental groups on the hypothetical donation amount. From this, we can conclude that our experimental stimulus failed to prime participants on SDO. However, SDO and RWA were significantly associated with donation amount, but CDO was not. Furthermore, as a result of this study, some other interesting data has come to light. Firstly, participants differed by sex on the percentage of students they believed that used student support services, with women believing that more used them than men. In addition, participants of different ethnicities differed on how effective and important they believed student support services were, with minorities such as Māori and those who fall under 'Other' generally considering them more effective and important that Pākehā participants.

In addition, we have found further support for $\mathrm{SDO}_{7}$, both by finding further evidence to support the idea that SDO is a four-factor model, but also by confirming that it works as intended in another culture. We have also investigated CDO, and found evidence that it is not as reliable a measure as $\mathrm{SDO}_{7}$, though it does seem to be a twofactor model, as predicted. We also found a significant effect of CDO on how effective student learning services can be, as well as a significant effect of SDO and CDO on how important participants perceived such services to be. We have also found more evidence for the idea that SDO is not a fixed personality trait, given that it seemed to differ from pre-test to post-test, despite the fact that the priming mechanism did not seem to work as intended. It is worth nothing, however, that the differences we observed, insignificant as they were, were in the direction we hypothesized they would be. Also, RWA and CDO appeared to be more fixed, as there was no significant difference between pre-test and post-test scores for them. 
However, there is something that may need to be considered when using $\mathrm{SDO}_{7}$ in future studies. In this study, the correlation between $\mathrm{SDO}_{7}$ and RWA was reported to be 0.61 in the pre-test and 0.64 in the post-test. These correlations are much greater than past studies using earlier versions of the SDO measure alongside RWA measures. For example, Sibley and Liu (2004) reported a correlation of 0.56 when using an older version of the SDO measure (Pratto, Sidanius, Stallworth, \& Malle, 1994) with a sample consisting mainly of Pākehā undergraduates similar to this study. The possibility that the $\mathrm{SDO}_{7}$ measure may correlate with other variables in a different manner than past versions cannot be denied. This could mean that larger, potentially significant correlations might be found where past measures detected none. Alternatively, it could mean that past correlations might not be found with $\mathrm{SDO}_{7}$. The only way to be sure would be to compare past versions of the SDO measure with $\mathrm{SDO}_{7}$. First of all, they should all be correlated with a measure of RWA drawn from the same sample, to test if this hypothesis that $\mathrm{SDO}_{7}$ is correlating differently with RWA compared to older SDO measures is accurate. Furthermore, all the versions of the SDO measure could be correlated among themselves, to see how much they actually differ from each other. Given that $\mathrm{SDO}_{7}$ was a reaction to criticisms over how definitions of SDO have changed over time (Rubin \& Hewstone, 2004), it might be worth investigating how much they actually differ, to see if these concerned were well-founded.

Furthermore, it appears that perceived effectiveness and importance of student support services was significantly correlated with perceived effectiveness of the advertisement stimulus, with those who believed that such services were effective and important believing that the advertisement would be more effective than those who did not believe that such services were effective or important. Furthermore, SDO and RWA were negatively correlated with perceived effectiveness and important of student services, while CDO was positively correlated with them. Indeed, regressions revealed that a combination of all three explained some of the variance in effectiveness, while SDO and RWA together without CDO best explained the variance in importance. All three also seemed to predict variance in hypothetical donation amounts, but only SDO alone came anywhere close to being able to significantly predict scores. 


\section{Implications and future studies}

\section{Improving on this study}

This study offers plenty of food for thought on how this area of research can be advanced in future. First of all, there is the possibility that our advertisement manipulation was not sufficiently true to life, and could not produce the increase in SDO hypothesized. Typically, the advertisements used by charities in real life are far more complex and detailed, using video and audio to convey their message, rather than the text and static photograph used in this study. If this is the case, then our failure to prove our hypothesis could be the result of methodological error, as without a manipulation that fails to properly emulate a real life scenario, we would not see the rise in SDO we hypothesized would come about. As a result, we would not see the effects we hypothesised would come about.

One way to test this theory would be to replicate the study, but instead of a still advert, we use adverts that were actually used in reality. Because these are real adverts, we would be properly emulating the conditions in which we believe the increase in SDO would occur outside of the lab. However, some facts must be taken into consideration in replicating the study in this way. First of all, one would need a control advert, and an experimental advert. Because our hypothesis holds that adverts that make social standing and hierarchies more salient will produce the proposed increase in SDO, the experimental advert would need to depict a crisis revolving around those low on social hierarchies. Many such adverts exist, such as the previously mentioned pleas for donations in response to the 2014 Ebola outbreak in West Africa.

However, one would have to find a similar advert to properly avoid any chance of a variable that was unaccounted for influencing the result. Finding such adverts for donations in response to an epidemic that is striking first-world nations would be difficult. Proper medical care and vaccination renders such epidemics unlikely to strike those in the wealthier parts of the world. To avoid this problem, I would recommend using charitable adverts concerning natural disaster relief instead. Because natural disasters strike at random, they can impact the first world just as easily as the third world. As an example, using the 2004 Indian Ocean earthquake and tsunami (Lay et al., 2005) as the experimental stimulus and Hurricane Katrina (Knabb, Rhome, \& Brown, 2005) as the control stimulus. Both disasters involved massive flooding and loss of life, 
but Katrina struck a first-world nation while the Indian Ocean earthquake damaged multiple less wealthy nations.

Using advertisements from charities to donate towards the relief effort for these two disasters, one may be able to sidestep the issue of confounding variables while keeping the stimuli as similar as possible. However, there is another issue that is harder to account for. It is possible that one of the variables that may influence any SDO priming that such adverts cause could be the time since the issue presented in the advert happened. If this is true, using archived advertisements may not be enough to fully imitate the real-life scenario that we envision. Using more recent advertisements could avoid the issue, but still represent the challenge of finding another recent advert as the control stimulus that is similar but less demographically and hierarchically charged would prove difficult.

Conducting such a study would answer the question raised by this study once and for all; do advertisements that highlight the suffering of those low on social hierarchies prime SDO in those higher in the social hierarchy? Because it is using actual adverts, it would have the strongest external validity possible. Thus, the results from it would be difficult to refute.

Further research on the findings of Huang and Liu (2005)

Furthermore, there is the possibility that our priming mechanism simply was not priming SDO in the way that Huang and Liu (2005)'s study did. Thus, the idea that priming SDO may impact charitable intent is still possible. Judging by the negative correlation we found between SDO and charitable intent, this is quite likely. A future study in which the priming apparatus is a translated and adapted version of what Huang and Liu (2005) used could be done that replicates this study in every other way. Such a study may show a significant difference, both within a group from pre-test to post-test and between groups, of which only one was primed on SDO. If this is found, then it is evidence for the idea that SDO is plastic across cultures. And should the primed groups show less charitable intent than the control group, or perhaps even themselves from a past study, that supports the idea that priming SDO will reduce charitable intent.

It could be possible that something native to Taiwanese culture that making the priming of SDO possible. If this is the case, then their results may not be generalized to 
other cultures. But what could this aspect of Taiwanese culture be? One possibility is a difference in how they perceive hierarchy. Zhang, Lin, Nonaka, and Beom (2005) investigated how endorsement for Confucian values differed among the young in four East Asian nations; Japan, China, Taiwan and Korea. Specifically, they looked at the Confucian values of interpersonal harmony (solidarity and tolerance of others), relational hierarchy (respecting ones superiors, obedience towards established social hierarchies) and traditional conservatism (moderation, having few desires).

Their results suggested all of these values were keenly felt by the young, even in a nation that is slowly undergoing globalization and the introduction of new ways of thinking. The important thing to take away from this is that a philosophy that emphasises, among other things, understanding hierarchy is deeply seated in Taiwanese and East Asian culture (Xu, 1998). As a result, it could be possible that the priming effect observed by Huang and Liu (2005) can only occur within cultures where the concept of social hierarchy is heavily embedded in one's culture. Although it could be argued that all cultures endorse hierarchies to some degree, differences in mean SDO scores by nation have been observed (Pratto et al., 2000). As SDO is a measure of endorsement of social hierarchies (Sidanius \& Pratto, 1999), nations with lower average SDO scores would endorse hierarchies less and thus, presumably differ from Taiwanese culture in this regard. Furthermore, if we take this possibility onto consideration, along with the above issue as to what exactly Huang et al. had primed, the possibility that this study failed to replicate the priming effects they observed becomes more feasible.

\section{Implications if our findings are accurate}

Next, let us consider the implications of these findings if we take them at face value. First, assume that charity advertising really does not prime viewer's SDO. This could mean that priming people on SDO, as Huang and Liu (2005) did involves more than simply making salient the differences between groups in a social hierarchy. One possibility is that they primed the participants with surveys and stimuli that do not commonly occur outside of the laboratory, while we presented participants with a more natural stimulus, something they might see in real life. It is possible that the more 'unnatural' manner of presentation done by Huang and Liu (2005) could be a contributing factor to the priming effect. An open letter by Kahneman (2012) has pointed out that, in recent times, priming effects in psychology have come under attack 
due to a lack of external validity. Attempts at replicating past findings have failed to do so, which has cast a suspicious pall over the whole practice. Thus, it could be possible that the results seen by Huang and Liu (2005) fall among other priming experiments that are difficult to replicate.

Also, the difference between the priming of group membership and the priming of intergroup differences must be made clear. To prime group membership implies reminding someone of all the personal traits that makes them part of a collective, the ways they are the same as others. To prime intergroup differences, meanwhile, implies reminding someone of all the ways they differ from another group of people. These are clearly separate things, even if they might be interlinked to some degree. As such, it is possible that one of these priming methods does have an influence on charitable intent, while the other does not. Briley and Wyer Jr. (2002) found that by priming participants on their cultural identity, they would be more likely to make decisions that minimized negative outcomes for themselves and those who fell within their same cultural group. Charities typically aim to prevent negative outcomes, so one could argue that priming people on their cultural identity will make them more likely to donate to a charitable cause that benefits their ethnic group.

\section{SDO and altruism}

Now that we have evidence that SDO impacts negatively with charitable intent, the idea that donating to charity is done to increase social standing seems suspect. Being high in SDO appears to have a negative correlation with wanting to donate to charity, but SDO is also the degree to which one believes in social hierarchy. Hypothetically, being high in SDO would make increasing one's social standing seem more desirable, but that would also decrease any intent to donate to charity. Consider the findings of Milinski, Semmann, and Krambeck (2002). They proposed that a public donation to a charitable cause could be rewarded by an increased standing with those in one's social group. Their study found that publicly made donations came with rewards in the form of increased monetary income from others in the social group, as well as an increase in political affluence, as those social others elevated the donor to represent them. The latter reward could be seen as desirable by those who are high in SDO, as it puts them in a higher position in a hierarchy. But our results would suggest that they would be less likely to donate because they are high in SDO. 
A possible reason for this apparent conflict is that 'publicity of donations' may be a mediating factor between SDO and intent to donate to charity. One way of testing this hypothesis is to have participants complete measures of SDO and then be randomly assigned to one of three conditions. The first condition is a control condition and simply gives the participant the chance to donate towards a charitable cause. The second condition would give participants the same option, but they would be told that all who donate would have their names displayed at an upcoming function for the charity. This would be the 'low publicity' condition. The third condition would allow participants to donate, and they would be told that all donors would have their names read aloud to a gathered audience at the upcoming function. This would be the 'high publicity' condition. If this hypothesis is correct, we should see that participants who score highly on SDO are more likely to be willing to donate in the high publicity condition compared to the low publicity and control condition. A test for moderation should also reveal that publicity has a mediational effect on the relationship between SDO and intent to donate, with greater publicity reducing the influence SDO has on intent.

In addition to this, it could be the simple fact that donating to charity can make one feel good about oneself. Returning to the findings of Moll et al. (2006) concerning the possibility that charity is done because it activates the mesolimbic reward pathways in the brain, it could be said that those who are high in SDO could still be willing to donate to charity simply because doing so activates these pathways, resulting in a positive feeling. This could lead to an investigation as to whether or not the desire to feel pleasurable feelings can override any opposition to helping others lower in hierarchy in those who are high in SDO. The best way to test this hypothesis involves brain scans, so knowing the regions of the brain that are strongly correlated with SDO is an important first step. Chiao, Mathur, Harada, and Lipke (2009) seemed to identify that preference for social dominance seems to be correlated with activation in the left anterior insula and anterior cingulate cortices. They tested this by having participants in an fMRI machine view images of people in pain as well as neutral images of people as a control. At each image, participants had to rate how much empathy they felt for that person. Thus, a potential future study would be to look at how activation in the mesolimbic reward pathways differs between people of high SDO and low SDO in response to a chance to donate towards a charitable cause. 
A study to test this hypothesis could be done using a similar setup, in which participants would view images of people in need of charitable aid alongside neutral images, with participants being asked how willing they would be to donate towards a charity that would help them. As mentioned previously, the issue of hypothetical bias may come up, so explaining hypothetical bias and asking people if they would be willing to donate ten dollars specifically could sidestep this problem (Brown, Ajzen, \& Hrubes, 2003). Should this hypothesis be correct, we should see more activation in the left anterior insula and anterior cingulate cortices in people with high SDO compared to those with low SDO. However, if they still say they would donate to charity, we should also see activation in the mesolimbic reward pathways. We may also see activation in the subgenual cortex and the septal region if the participant would donate out of genuine desire to help (Moll et al., 2006). If the innate desires to feel good does to some degree mediate the relationship between SDO and charitable intent, we should see more activation in the mesolimbic reward pathways than in the left anterior insula and anterior cingulate cortices when participants high in SDO are offered the chance to donate towards a charitable cause.

\section{SDO, RWA, and altruism}

This study has given us evidence that SDO and RWA correlate negatively with intent to donate to charity. However, the precise mechanism of such a relationship remains unknown. Drawing on past research into perceptions of charity and welfare, we may be able to find a possible pathway. For example, Petersen, Slothuus, Stubager, and Togeby (2011) conducted research into public opinion on welfare policies. They hypothesized that people have a 'deservingness heuristic' that activates when people consider welfare programs, making them think in terms of people who need help. They also claimed that social class would have no impact on whether or not this heuristic will activate. They found evidence for this hypothesis from data collected from two studies. If people do have a deservingness heuristic that works when they must consider giving money to those in need, the perhaps the same heuristic is activated by the opportunity to donate to charity.

This could tie into the idea of legitimizing myths (Sidanius \& Pratto, 1999), as the idea that 'those without the advantages I enjoy deserve my help' can be perceived as a HA legitimizing myth. Given Petersen et al.'s hypothesis that this is a heuristic that 
everyone has, it would likely have a high degree of consensuality. This could also be a manifestation of the 'just world' theory (Lerner, 1980), as to donate towards the deserving is an act that would make the world more 'just.' Furthermore, such a heuristic would go contrary to system justification theory (Jost, Banaji, \& Nosek, 2004), which holds that people desire to justify the status quo when it benefits them, even if it is disadvantageous to others. To consider others deserving of help that might go against the status quo, one must overcome system justification.

If this is the case, we can argue that such a heuristic would be more likely to decide that people who need charity are more deserving than people who need welfare. Charities, in general, put money towards those with disabilities or those suffering in the wake of natural disaster. People may be more likely to decide that those people need help compared to those on welfare programs, some of whom may be perceived as being able to support themselves, but are not.

How could we test the idea that such a heuristic also influences the decision to donate to charity? In the study of Petersen, Slothuus, Stubager, and Togeby (2011), they had participants answer a question over the phone regarding whether or not restrictions on welfare should be made more stringent for people similar to an example of a person on welfare. The example would differ between participants, from a healthy young man to an elderly woman with a work-related injury. A similar method could be used in a study to find support for the idea that a deservingness heuristic also applies to donating to charity. Instead of making the question about welfare restrictions, participants could be asked how likely they would be to support a charity that helps people like an example given, with each example varying in how deserving they would be of such help. However, there could be an effect of hypothetical bias in such a study, the problem of people claiming they would pay more than they actually would given an actual chance.

A study by Brown, Ajzen, and Hrubes, (2003) offers a way to avoid this. By informing participants of the issue of hypothetical bias, it helps to eliminate effects of it in their hypothetical answer, especially if they are being asked to donate a high amount (eight to ten dollars showed the greatest reduction in the influence of hypothetical bias in their study). As such, this study could specifically ask if they would donate ten 
dollars towards such a cause after being informed of hypothetical bias and asked to try and avoid it.

For example, participants could be presented with one of four scenarios in they are asked if they would donate ten dollars towards a charity that supports a hypothetical individual, with each scenario having a different individual. Each individual would vary in terms of how much they 'deserve' to have a charity for them. To go one step further, a 4 x 4 study could be conducted, using the four examples of welfare beneficiaries along with the four charity scenarios just presented. Participants would be presented with one welfare scenario and one charity scenario, and asked to rate which one is fairer and more deserving. This way, we may be able to find whether or not people find welfare cases or charity cases more deserving of the aid they get. Theoretically, if the deservingness heuristic does influence the decision to donate to charity, we should see that participants are less willing to donate towards a charity that is seen as supporting less deserving causes compared to charities that would support more deserving causes. Furthermore, this study could have participants fill in a measure of SDO and RWA. This way, we could test to see if such scenarios interact with SDO and RWA in some way. If they are found to interact, this could be interpreted as evidence for the idea that the deservingness heuristic plays some role in how SDO and RWA impact on intent to donate to charity.

Furthermore, there is the possibility that the attempted priming of the participants could have been flawed if the 'deservingness heuristic' is the mechanism through which SDO and RWA influence charitable intent. Petersen, Slothuus, Stubager, and Togeby (2011) found that the 'deservingness heuristic' activates when discussing and deliberating over welfare. The adverts used in this study only spoke of the merits of the organizations mentioned, with no comments on how it would be a worthy cause to donate money towards. The prompt for the public goods game similarly did not attempt to bias the participants towards donation, nor did it discuss what any donated funds would go towards. As such, it could be possible that this minimum discussion of charity was not sufficient to activate the deservingness heuristic. Future studies could bring up the benefits of donating in the stimulus, or have participants engage in a discussion on charity beforehand to ensure that the heuristic is activated. 
Greater understanding of this deservingness heuristic would greatly benefit charities, as they would be able to utilize it more efficiently gather donations. By presenting potential donors with some kind of stimulus that engages the heuristic and makes the donor decide that the charity deserves enough, they can increase the amount of donations they receive.

\section{Empathy and altruism}

Could empathy possibly act as a mediator of this correlation between SDO and charitable intent? The participants were all university students participating for course credit, and the money put into the public pool would have gone towards services that help other university students. The fact that the recipients of the donated pool money were similar to the participants may have increased the participant's empathy towards them (Halpern, 1955).

In addition, Sidanius et al. (2013) displayed that empathy and SDO had a twodirectional longitudinal relationship, with SDO measured at one time having a negative relationship with empathy measured at a second time. This does seem to support the idea that high empathy caused by similarity could have influenced the outcome of this study. But Sidanius et al. (2013) also found that SDO seems to have a stronger influence on empathy than empathy does on SDO. As a result, this argument more supports the idea that SDO has no influence on willingness to donate than it does the idea that SDO does affect willingness to donate, but it simply was not adequately primed in this study. Considering that we seemed to find that SDO did have a negative relationship with charitable intent, it may be said that this is not the case. However, these studies were longitudinal, and that may be the key difference.

The first potential follow-up to this study would be to establish if any sense of empathy borne from similarity had any impact on the results. One way of doing this could involve repeating the study with three new conditions. In the first condition, participants are given the chance to donate towards a cause that benefits those similar to themselves. For example, if the sample is drawn from university students, the recipient of the donations would offer some benefit to other university students. This is the control condition, which closely replicates the earlier study. The second condition would give participants the chance to donate towards a cause that would inspire 
empathy in most people, but not because of any feelings of similarity with those the donations would benefit.

One example could be a charity that benefits the mentally disabled, or those who have lost a limb. Most people would still feel empathy for such a cause, but would not be similar to them, now nor in future. The point of this condition would be to test to see if empathy inspired by similarity differs in any way from empathy born of pity or sympathy. Finally, the third condition would give participants a chance to donate towards a cause that they may not particularly care about one way or the other. For example, if we imagine that the student sample was drawn from the psychology courses, the cause could be a dinner party for one of the other schools at the university. It must not be a cause that would inspire any kind of negative affect, as that would be a confound in the study.

If empathy does have any influence on the effect of SDO on charitable intent, we should see a difference in the amount donated between the empathy groups and the no empathy group. Secondly, if empathy inspired by similarity is different from other forms of empathy in how it influences SDO and charitable intent, we should see a difference between the empathy-by-similarity group and the empathy-without-similarity group.

Furthermore, if empathy really does have any effect on the influence SDO has on willingness to donate to charity, a study similar to the one done by Sidanius et al. (2013) could be done to test it. As their results suggest that the interaction between SDO and empathy is longitudinal, this study would also need to be longitudinal. At the first stage, participants would be tested on SDO measures, as well as Davis' (1983) 7-item Empathetic Concern sub-scale. The point of this first stage is to identify participants who score low in SDO and high in empathetic concern, as they will form the sample group of the next stage of the process. These participants will be primed in SDO and the complete the same scales again before being given the opportunity to donate towards a charitable cause.

Hypothetically, because these participants scored high in empathetic concern, but have had their SDO temporarily increased, they should have both high SDO and high empathetic concern simultaneously. As a result, if they are still willing to donate towards the charitable cause despite temporarily heightened levels of SDO, we can 
assume that empathy can override SDO when it comes to charity. However, there is still the possibility that priming people on SDO may actually reduce their empathy, which would be an important discovery in and of itself.

The findings of this study would be beneficial in many ways. First of all, should empathy actually manage to block negative influences on willingness to donate that would be useful information to all organizations that run off donations. By employing methods that increase empathy, they can reduce the impact of SDO on donating behaviour, potentially increasing their income. But if it is found that empathy actually decreases when SDO is primed, this could potentially open up a new path in empathy studies. By having a means of temporarily reducing empathy, research into sociopathic behaviour may become more viable without requiring actual sociopaths, for example.

\section{Morality and altruism}

Another factor that must be considered is personal moral codes. Like empathy, there is a possibility that they could somehow interact with the relationship between SDO, RWA and charitable intent.. Wilson (2003) found startling information as to the interplay of SDO and personal moral codes. Namely, while he found evidence that SDO has a consistent negative relationship with idealism, the belief that actions should not harm others, it did not show any consistent relationship with relativism, the idea that actions cannot be compared in a moral sense. This is evidence for the idea that there is interplay between personally held moral codes and SDO. As we did not attempt to measure the moral codes of participants, it is unknown as to whether or not this could have been a factor. Furthering this argument, there is also evidence that such moral codes also influence willingness to donate towards a cause. Van der Linden (2011) found evidence that personal moral codes can strongly influence personal decision to donate towards a charitable cause. Indeed, it is responsible for a large amount of variation in such a decision. As such, it can be easily argued that such variables could have had a major impact on this study.

Moral codes can be difficult to measure and define, but van der Linden (2011) was able to utilize the extended Theory of Planned Behaviour model (Smith \& McSweeney, 2007) to create a questionnaire that incorporates it. To test the idea that personal moral codes can moderate the effect SDO has on intention to donate towards a charity, we would need a two-part study. The first part would simply involve having 
participants complete the Theory of Planned Behaviour questionnaire van der Linden devised, so as to identify participants with the highest levels of moral norms. These participants would be invited back and split into a control group and an experimental group. The experimental group would be primed in SDO and invited to donate towards a charitable cause, as will the control group.

If high levels of moral norms really do mediate the relationship between SDO and charitable intent, then we should still see roughly similar amounts donated from both groups, in spite of the experimental group being primed in SDO. We must also consider the fact that the amount participants said they would donate was purely hypothetical. There has typically been a difference in the amount of money people say they would donate given the chance, and the amount they actually donate when that chance occurs (Champ \& Bishop, 2001). As a result, we can hypothesize that different factors may have different effects on hypothetical donations and actual donations.

As an example of such a factor having different levels of effects, consider the findings of Brown, Champ, Bishop, and McCollum (1996). They investigated the effects of response format on willingness to pay in both hypothetical donating scenarios and actual donating scenarios. Their results indicated that under hypothetical scenarios, the response format had a much larger impact on willingness to pay than real scenarios, with a closed, dichotomous choice yielding much higher levels of willingness. As our study consisted of an open choice with the option to pay nothing being genuinely tempting, there is the possibility that willingness to pay was affected in a manner we did not intend.

Furthermore, let us consider moral signatures and the MFT once more. Milojev et al., (2014) found that RWA was correlated with the High Morality moral signature, a high level of endorsement in all moral foundations they measured. And yet, we find that RWA correlated negatively with charitable donations, something typically considered a moral action. Could this mean that their findings only hold in a hypothetical sense, and when placed in a genuine moral dilemma, different results might be observed? Obviously, this would be impossible to test, as no ethics board would permit placing participants in even a facsimile of a genuine moral dilemma.

However, let us consider the moral foundations that are present in MFT. Care/harm, fairness/cheating, loyalty/betrayal, authority/subversion, and 
sanctity/degradation (Graham et al., 2012). Which of these would acts of charity fall under? One could make an argument for care/harm, as donating towards a cause is a way of showing how much one cares. But it could also be said to fall under fairness/cheating, as donating towards a cause that helps the less-fortunate can be seen as balancing the scales in their favour. Alternatively, it could fall under loyalty/betrayal, as it can be interpreted as showing solidarity to your fellow man by supporting their cause. Thus, an act of charity can be seen as falling under multiple foundations. Ergo, we must ascertain which moral foundations influence charitable intent before continuing this train of through.

A study by Winterich, Zhang, and Mittal (2012) looked at charity and MFT. Their findings suggested that when a charity's moral foundations match with a potential donor's political identity, as evidenced by their mission statement or managerial style, the donation amount increases. This does not answer the question as to what foundation charity tends to fall under, so a new study would have to be conducted to ascertain that. Using the Moral Foundations Questionnaire (Graham et al., 2011), we can measure the degree to which people endorse the moral foundations. Then, participants would be offered a chance to donate to a charitable cause. Great care must be taken at this stage. As Winterich et al. found, the moral foundations of a charity can influence the likelihood of someone donating to them if they closely match their own political preferences. Not mentioning a charity by name or their function may be the best way to get around this. We should be able to observe positive correlations between the moral foundations that dictate charitable intent and the amount donated. It would be difficult to predict exactly what foundations that charitable intent is correlated with. Consider again the findings of Milojev et al. (2014). RWA was correlated with High Moralists, those who rated all five moral foundations highly. SDO was correlated with Neutral moral signatures, which consisted of all five moral foundations being rated low. And yet, both variables have the same negative correlation with charitable intent. Thus, hypothesizing what foundations charitable intent would be strongly correlated with would be difficult.

Findings from such a study would be massively invaluable to charity organizations. By knowing the moral foundations that charitable intent is rooted in, they can develop targeted advertisement campaigns that attempt to trigger these foundations, potentially increasing the amount of donations they draw. Furthermore, it would 
advance our understanding of altruism, a facet of humanity that has been argued over at great length throughout the ages. It would mean a deeper understanding of exactly what drives people to go out of their way to help others at their own expense.

\section{Ethnicity and altruism}

Those who donate to charity are typically looked on favourably, and doing prosocial deeds typically makes one feel good about oneself. The opposition of public policy is typically done privately as one's role as a voter, if they get a say in it at all. One final possibility is the influence of 'white guilt,' the idea that Pākehā still has a debt to pay for the prejudices and actions perpetrated by the European settlers against the Māori. This could override any influence that SDO could have on one's decisionmaking. As the majority of our participants were Pākehā, to the point that we felt it best to remove participants of other ethnicities from our analyses, this is a valid follow-up to further investigate our findings.

As an example of how collective guilt can drive attitudes towards indigenous minorities, Brown, González, Zagefka, Manzi, and Čehajić (2008) investigated how feelings of collective guilt and collective shame influenced attitudes towards reparation in indigenous Chileans when the target was the Mapuche, Chile's largest indigenous group. The results indicated that both collective guilt and collective shame had an impact on feelings towards reparation, with guilt having longitudinal effects and shame moderating that effect. A similar effect may be taking place in New Zealand with NZ Europeans/Pākehā experiencing a similar effect towards the Māori.

In the case of charitable causes towards minorities, there is a possibility of some kind of collective guilt or shame impacting how the majority feel about supporting such a cause. To test this, we would first need to measure how keenly participants feel such things. Brown, González, Zagefka, Manzi, and Čehajić (2008) devised a measure for doing so, but it would first need to be adapted to the relevant majority and minority people for the culture this hypothesis is being tested in. Obviously, such a study can only be conducted in a cultural context where the majority and minority have come into conflict in the past, such as Pākehā and Māori, or white and black Americans.

Participants would all need to be from the majority who are high on the social hierarchy. For example, in New Zealand it would be Pākehā. 
They would first be tested on collective guilt and shame, as well as SDO and RWA. Then, participants would be given the opportunity to donate towards a minority cause. Obviously, results may differ between cultures depending on multiple factors. For example, cultures in which official reparations on behalf of the government towards minorities over past tragedies are being paid may result in the majority having less collective guilt overall, compared to nations where no such reparations are being made. But, if collective guilt and shame do interact with SDO and RWA when it comes to opportunities to support minorities that are the source of that guilt and shame, we should see some kind of interaction in subsequent analyses of the data.

Looking back at the apparent effects of ethnicity on perception of student learning services, the question becomes 'why does this occur?' Minority participants appeared to think that such services were both more effective and important that Pākehā participants. One possible reason for these results could be the fact that such services, especially those targeted at a single group such as Te Rōpū Āwhina, are examples of hierarchy-attuning forces. They provide help with study and learning to groups who may oftentimes struggle in such areas, allowing them to equalize with other groups who do not have to deal with such issues. New Zealanders seem to have an implicit belief in biculturalism (Sibley \& Liu, 2007) which means that the idea that 'Māori and Pākehā deserve an equal chance' could be a legitimizing myth with strong embeddedness in New Zealand culture. This could explain why we generally saw more positive attitudes from minority groups on the effectiveness and importance of programmes. When primed with adverts concerning Te Rōpū Āwhina, that particular legitimizing myth may come to mind across all ethnicities that identify as 'New Zealanders', and so shapes opinions accordingly, with the only difference being the extreme to which their opinions shift.

Another aspect of participants that must be considered when thinking in terms of legitimizing myths, however, is that all participants were university students. As they are all undergoing further education, they may all be invested in the legitimizing myth of 'all people deserve a good education,' a myth that would have a high consensuality, embeddedness and certainty amongst university students. This could also explain why we saw increased belief in effectiveness amongst all ethnic groups. 
Making a study surrounding how legitimizing myths may be influencing attitudes towards minorities would be very difficult, as the researchers would first have to identify what legitimizing myths are the most pervasive in their culture. The only way this could be done is through an extensive qualitative study, requiring interviews of people from all over the nation. In the interviews, participants would need to be asked why they think stereotypes about minorities and majorities exist, as well as why they think that beliefs that pervade society exist. From this vast swath of data, answers that fall under similar themes would need to be combined to identify the legitimizing myth behind it. Once all the data has been analyzed, the researchers should have a list of legitimizing myths of their culture.

From this, they can create a questionnaire concerning those myths, assigning a Likert scale to each one asking whomever is taking it how much they agree with each myth. Then, the actual study can start. Participants would be measured in their endorsement of legitimizing myths in the first study, with those scoring who score low in myths that demonize the minority and high in myths that endorse egalitarianism being the core focus of the second study. Such participants would be primed in SDO and asked to donate towards a charitable fund that would help minorities. If endorsement of hierarchy-attuning legitimizing myths and rejection of hierarchyenforcing legitimizing myths mediates the relation between SDO and intent to donate to charity, then we should see participants in the experimental group donate money towards the cause at least on the same level as a control group.

We also found a difference between ethnicities on attitudes towards student learning services. In general, minorities appeared to feel that such services were more effective and important that Pākehā participants. For the Māori students, one reason this could be is that they have such services made only for them. Having an exclusive group may somehow result in a more positive opinion in such practices. As for participants on the 'Other' category, they may be foreign exchange students, and their higher opinions could be due to the acculturating processes that such support services create. According to Berry (1997), acculturation is the process by which cultures change by coming into contact with other cultures. From such meetings, a fourfold model emerges made of two dimensions that a culture must make. The first is the degree to which they wish to maintain their own culture, and the second is the degree to which they want to exist alongside the other culture. For foreign exchange students who are new to New Zealand 
and need help fitting in, such services would be a great boon, allowing for improved understanding of both New Zealand as well as the university experience.

\section{Limitations of the present study}

The first, major issue that our present study suffers from is the relatively small sample pool. The fact that a huge majority of participants were NZ European/Pākehā especially hurt it, since it was such an ethnically concerned topic. A greater sample of Māori would have made it a stronger study, and given us the ability to draw firmer conclusions as to the effects of ethnicity on charitable donation, as well as any interaction SDO could have had with it. In addition, all participants who were neither Pākehā nor Māori were lumped into an 'Other' category, which may have resulted in us losing some degree of specificity that could have been important.

Also, this study faces the same issue that many modern psychology studies face, and that is the limited age range of the participants. Using psychology undergraduate students as subjects in exchange for mandatory course credit allows for easy access to sizable sample pools, but it does mean that a majority of participants fall into the same categories. As mentioned above, a majority of them were NZ European/Pākehā, the most common demographic to enter tertiary education in New Zealand, but also a majority of participants fell into the $18-20$ age range. This is obviously a very narrow slice of potential age ranges, which may be a contributing factor towards several variables investigated in this study. Such links could have gone unnoticed simply because there were not enough people in different age categories for such views to be properly represented.

It should also be noted that a majority of participants were women. Given that this study investigated SDO, a factor repeatedly shown to be influenced by sex (Pratto, Stallworth, \& Sidanius,1997) (Sidanius, Pratto, \& Bobo, 1994). Having such a sample skewed in favour of the sex that commonly shows less SDO could have influenced the data in various ways, such as making the mean SDO scores lower. A future study would absolutely have to have more thorough representation across age and sexes.

Another challenge that we faced was that there was no time to properly test the priming materials. As such, we were not sure if the advertisements would actually be priming SDO or not. Obviously, this is a major flaw that would need to be corrected in 
future, either by taking the time to test them first, or by replacing them with more established, valid SDO priming materials, such as those used by Huang and Liu (2005).

The fact that the study was conducted online brings with it a share of issues. The most immediate issue is that we cannot control the participant's environment as they complete the study. There could be a myriad of factors that influence how they answered the study, and we have no idea whether or not to account for them. This is a known issue with distributing surveys online, and while it could be argued that this gives them greater external validity by having it take place outside of the laboratory, it also has its downsides. They could be distracted by something else as they were filling in the question, resulting in half-thought-out answers. They could have completed it early in the day or late at night, so tiredness could be a factor. Participant mood, immediate environment and many other factors are not accounted for. The first of which is the fact that the public goods game was not conducted with a group as it usually is. The reason public goods games can give incentive towards being selfish is that someone does not know how much money someone else is planning to put into the pool. As such, being selfless and donating all your money can result in net loss because everyone else was selfish and kept theirs. Because the participant was not in a group of others also participating in the study as they completed it, they would actually have more incentive to donate all the money into the public pool, as it would be doubled and returned to them. Even with the portion going to the student support service, that would still likely mean more money for them.

Another issue with the study is relating to the public goods game is the fact that it is only a hypothetical scenario. We were not able to use actual funds for participants to keep or donate as they would like. This is an issue, as past studies have indicated that the amount of money people claim they would donate and the amount of money they actually donate can end up being wildly different (Brown \& Taylor, 2000). In addition, public goods games are typically played in groups. However, because this study was administered via an online survey, each participant was essentially completing it by themselves. This could be a potential confound. However, there is an additional issue that only comes up when conducting a study such as this in New Zealand. Sibley and Liu (2004) found evidence that NZ Europeans/Pākehā who were high in SDO would show support for symbolic bipartisan policies (i.e. policies that would not interfere with 
their lives) but would show less support for resource-specific bipartisan policies (i.e. policies that might tax them or put them in competition with Māori).

As this study involves SDO, these findings become a concern. Because the amount the participants would donate was purely hypothetical, it could have been perceived as a symbolic gesture, and thus, any influence SDO may have had on it would be lessened. A future study could use an actual public goods game with actual funds to test this. Should participants who are high in SDO, either through priming or innately high be less willing to donate to a public pool where a portion of the money goes towards minorities, this hypothesis is supported.

But, this does raise another issue with this study. Namely, that the results are not terribly generalizable across different cultures. Sibley and Liu (2007) found evidence for the idea that New Zealanders are implicitly bicultural in a way that is not seen in other cultures. When presented with faces of Pākehā and Māori, both Pākehā and Māori rate them both as equally indicative of a typical New Zealander, suggesting an implicit attitude of biculturalism that is not seen in most other cultures. They compared this with a US sample, in which white faces were seen as more indicative of a typical American over African American faces. As such, we can conclude that, because this study tackles the issue of social hierarchies and multiculturalism within a nation that has a unique level of implicit biculturalism, it becomes difficult to generalize any findings to other nations where the population is less implicitly multicultural than New Zealanders.

\section{Conclusion}

One can argue the possibility that advertisements for charitable ventures may be unintentionally working to sabotage the efforts that the charity is making. Findings by Huang and Liu (2005) suggest that social dominance orientation is a variable that can be primed in people. Given that advertisements for charity typically emphasize differences in social standing between the viewer and the charity cases, viewers may be getting primed on social dominance orientation, which could reduce their willingness to donate. However, social dominance orientation is a factor in flux, due to recent criticisms aimed at it concerning definitions that have shifted over the years (Rubin \& Hewstone, 2004). As a result, this study first looked at new measures of SDO developed by Ho et al. (In print) and Pratto et al. (2012) in an attempt to discern which may be better for future research into social dominance orientation. Using confirmatory factor analysis, evidence 
was found that the multi-dimensional model previously believed to best represent social dominance orientation (Ho et al., 2012) fit the data best, while a two-dimensional model best fit the counter-dominance orientation data gathered. However, the newest social dominance orientation did show greater reliability than counter-dominance orientation, both in total, and the reliabilities of the separate dominance and anti-egalitarianism scales contained within. Subsequently, a new study was conducted using both scales, to test the effects of social dominance priming from advertisements on intent to donate towards a charitable cause. Participants either saw an advert for a student support service for all students, or an advert for a student support service specifically for minorities. They subsequently completed measures of right-wing authoritarianism, as well as the new social dominance orientation and counter dominance orientation scales. Afterwards, they engaged in a hypothetical public goods game, where a portion of the pool would go towards the service they saw the advert for. Subsequent analyses of the data suggested that our hypothesis was not supported. However, further analyses revealed other significant interactions, all of which were considered here. 
Tests of Social Dominance on Charitable Intent Towards Minorities

\section{References}

Ajzen, I. (1991). The theory of planned behavior. Organizational behavior and human decision processes, 50(2), 179-211.

Altemeyer, B. (1996). The authoritarian specter. Cambridge, MA: Harvard University Press.

Altemeyer, B. (1998). The other "Authoritarian Personality". Advances in Experimental Social Psychology, 30, 47-92.

Altruism. (2008). In J. William A. Darity (Ed.), International Encyclopedia of the Social Sciences (pp. 87-88). Detroit: Macmillan Reference USA.

Barclay, P. (2011). The evolution of charitable behaviour and the power of reputation. In S. C. Roberts (Ed.), Applied Evolutionary Psychology (pp. 149-172). Oxford University Press.

Bennett, R. (1997). 'They shouldn't let them out for us to see': Empathy and affect intensity as determinants of responses to representations of the facially disfigured in charity advertising. Journal of Nonprofit and Voluntary Sector Marketing, 2(3), 216-232.

Berdahl, J. L. (2007). The sexual harassment of uppity women. Journal of Applied Psychology, 92(2), 425.

Berry, J. W. (1997). Immigration, acculturation, and adaptation. Applied psychology, 46(1), 5-34.

Briley, D. A., \& Wyer Jr., R. S. (2002). The Effect of Group Membership Salience on the Avoidance of Negative Outcomes: Implications for Social and Consumer Decisions. Journal of Consumer Research, 29(3), 400-415.

Brown, K. M., \& Taylor, L. O. (2000). Do as you say, say as you do: evidence on gender differences in actual and stated contributions to public goods. Journal of Economic Behavior \& Organization, 43(1), 127-139. 
Tests of Social Dominance on Charitable Intent Towards Minorities

Brown, R., González, R., Zagefka, H., Manzi, J., \& Čehajić, S. (2008). Nuestra culpa: Collective guilt and shame as predictors of reparation for historical wrongdoing. Journal of Personality and Social Psychology, 94(1), 75-90.

Brown, T. C., Ajzen, I., \& Hrubes, D. (2003). Further tests of entreaties to avoid hypothetical bias in referendum contingent valuation. Journal of Environmental Economics and Management, 46(2), 353-361.

Brown, T. C., Champ, P. A., Bishop, R. C., \& McCollum, D. W. (1996). Which Response Format Reveals the Truth about Donations to a Public Good? . Land Economics, 72(2), 152-166.

Carli, L. L. (1999). Gender, interpersonal power, and social influence. Journal of Social Issues, 55(1), 81-99.

Champ, P. A., \& Bishop, R. C. (2001). Donation Payment Mechanisms and Contingent Valuation: An Empirical Study of Hypothetical Bias. Environmental and Resource Economics, 19(4), 383-402.

Chang, C.-T., \& Lee, Y.-K. (2011). The 'I'of the beholder: how gender differences and self-referencing influence charity advertising. International Journal of Advertising, 30(3), 447-478.

Chen, E. S., \& Tyler, T. R. (2001). Cloaking power: Legitimizing myths and the psychology of the advantaged. In A. Y. Lee-Chai, \& J. A. Bargh (Eds.), The use and abuse of power: Multiple perspectives on the causes of corruption (pp. 241261). New York, NY, USA: Psychology Press.

Chiao, J. Y., Mathur, V. A., Harada, T., \& Lipke, T. (2009). Neural Basis of Preference for Human Social Hierarchy versus Egalitarianism. Annals of the New York Academy of Sciences, 1167(1), 174-181.

Cortina, J. M. (1993). What is coefficient alpha? An examination of theory and applications. Journal of Applied Psychology, 78, 98-104.

Das, E., Kerkhof, P., \& Kuiper, J. (2008). Improving the Effectiveness of Fundraising Messages: The Impact of Charity Goal Attainment, Message Framing, and 
Tests of Social Dominance on Charitable Intent Towards Minorities

Evidence on Persuasion. Journal of Applied Communication Research, 36(2), 161-175.

Davis, M. H. (1983). Measuring individual differences in empathy: evidence for a multidimensional approach. Journal of personality and social psychology, 44(1), 113.

De Waal, F. B. (2008). Putting the altruism back into altruism: the evolution of empathy. Annual Review of Psychology, 59, 279-300.

Dictionary. (2015, June 25). Altruism | Define Altruism at Dictionary.com. Retrieved from Dictionary.com: http://dictionary.reference.com/browse/altruism

Downey, R. G., \& King, C. G. (1998). Missing Data in Likert Ratings: A Comparison of Replacement Methods. The Journal of General Psychology, 125(2), 175-191.

Duckitt, J. (2006). Differential effects of right wing authoritarianism and social dominance orientation on outgroup attitudes and their mediation by threat from and competitiveness to outgroups. Personality and Social Psychology Bulletin, 32(5), 684-696.

Duckitt, J., Wagner, C., du Plessis, I., \& Birum, I. (2002). The psychological bases of ideology and prejudice: Testing a dual process model. Journal of Personality and Social Psychology, 83(1), 75-93.

Fischbacher, U., Gächter, S., \& Fehr, E. (2001). Are people conditionally cooperative? Evidence from a public goods experiment. Economics Letters, 71(3), 397-404.

Forsyth, D. R. (1980). A taxonomy of ethical ideologies. Journal of Personality and Social Psychology, 39, 175-184.

Gatignon, H. (2010). Confirmatory Factor Analysis in Statistical analysis of management data. doi:10.1007/978-1-4419-1270-1_4

Gliem, J. A., \& Gliem, R. R. (2003). Calculating, interpreting, and reporting Cronbach's alpha reliability coefficient for Likert-type scales. Midwest Research-to-Practice Conference in Adult, Continuing, and Community Education. 
Tests of Social Dominance on Charitable Intent Towards Minorities

Graham, J., Haidt, J., Koleva, S., Motyl, M., Iyer, R., Wojcik, S. P., \& Ditto, P. H. (2012). Moral foundations theory: The pragmatic validity of moral pluralism. Advances in Experimental Social Psychology, Forthcoming.

Graham, J., Nosek, B. A., Haidt, J., Iyer, R., Koleva, S., \& Ditto, P. H. (2011). Mapping the moral domain. Journal of Personality and Social Psychology, 101(2), 366.

Halpern, H. M. (1955). Empathy, similarity, and self-satisfaction. Journal of Consulting Psychology, 19(6), 449-452.

Heaven, P. C., \& Bucci, S. (2001). Right-wing authoritarianism, social dominance orientation and personality: an analysis using the IPIP measure. European Journal of Personality, 15(1), 49-56.

Ho, A. K., Sidanius, J., Kteily, N., Sheehy-Skeffington, J., Pratto, F., Henkel, K. E., . . Stewart, A. L. (In print). The Nature of Social Dominance Orientation: Introducing the Social Dominance Orientation7 Scale. Journal of Personality and Social Psychology.

Ho, A. K., Sidanius, J., Pratto, F., Levin, S., Thomsen, L., Kteily, N., \& SheehySkeffington, J. (2012). Social Dominance Orientation: Revisiting the Structure and Function of a Variable Predicting Social and Political Attitudes. Personality and Social Psychology Bulletin, 38(5), 583-606.

Hu, L., \& Bentler, P. M. (1999). Cutoff criteria for fit indexes in covariance structure analysis: Conventional criteria versus new alternatives. Structural Equation Modeling, 6(1), 1-55.

Huang, L.-L., \& Liu, J. H. (2005). Personality and social structural implications of the situational priming of social dominance orientation. Personality and Individual Differences, 38, 267-276.

Jost, J. T., \& Thompson, E. P. (2000). Group-based dominance and opposition to equality as independent predictors of self-esteem, ethnocentrism, and social policy attitudes among African Americans and European Americans. Journal of Experimental Social Psychology, 36(3), 209-232. 
Tests of Social Dominance on Charitable Intent Towards Minorities

Jost, J. T., Banaji, M. R., \& Nosek, B. A. (2004). A decade of system justification theory: Accumulated evidence of conscious and unconscious bolstering of the status quo. Political psychology, 25(6), 881-919.

Jylhä, K. M., \& Akrami, N. (2015). Social dominance orientation and climate change denial: The role of dominance and system justification. Personality and Individual Differences, 86, 108-111.

Kahneman, D. (2012, September 26). A proposal to deal with questions about priming effects. Nature. Retrieved October 14, 2015, from www.nature.com/polopoly_fs/7.6716.1349271308!/suppinfoFile/Kahneman\%20 Letter.pdf

King, G., Murray, C. J., Salomon, J. A., \& Tandon, A. (2004). Enhancing the validity and cross-cultural comparability of measurement in survey research. American Political Science Review, 98(1), 191-207.

Knabb, R. D., Rhome, J. R., \& Brown, D. P. (2005). Tropical cyclone report: Hurricane Katrina, 23-30 August. National Hurricane Centre.

Lay, T., Kanamori, H., Ammon, C. J., Nettles, M., Ward, S. N., Aster, R. C., . . Sipkin, S. (2005). The Great Sumatra-Andaman Earthquake of 26 December 2004. Science, 308(5725), 1127-1133.

Lerner, M. J. (1980). The belief in a just world. Springer US.

Levin, S., Federico, C. M., Sidanius, J., \& Rabinowitz, J. L. (2002). Social dominance orientation and intergroup bias: The legitimation of favoritism for high-status groups. Personality and Social Psychology Bulletin, 28(2), 144-157.

Marwell, G., \& Armes, R. E. (1979). Experiments on the Provision of Public Goods. I. Resources, Interest, Group Size, and the Free-Rider Problem. American Journal of Sociology, 84(6), 1335-1360.

Milinski, M., Semmann, D., \& Krambeck, H. (2002). Donors to charity gain in both indirect reciprocity and political reputation. Proceedings of the Royal Society of London B: Biological Sciences , 269(1494), 881-883. 
Tests of Social Dominance on Charitable Intent Towards Minorities

Milojev, P., Osborne, D., Greaves, L. M., Bulbulia, J., Wilson, M. S., Davies, C. L., . . Sibley, C. G. (2014). Right-wing authoritarianism and social dominance orientation predict different moral signatures. Social Justice Research, 27(2), 149-174.

Moll, J., Krueger, F., Zahn, R., Pardini, M., de Oliveira-Souza, R., \& Grafman, J. (2006). Human fronto-mesolimbic networks guide decisions about charitable donation. Proceedings of the National Academy of Sciences, 103(42), 1562315628.

Mook, D. G. (1991). Why Can't Altruism Be Selfish? Psychological Inquiry: An International Journal for the Advancement of Psychological Theory, 2(2), 139141.

Morselli, D., Pratto, F., Zeineddine, F. B., Aranda, M., Stewart, A. L., Cidam, A., . . . Sweetman, J. (2012). Social Dominance and Counter Dominance Orientation Scales (SDO/CDO): Testing Measurement Invariance. International Society of Political Psychology, (p. 22). Chicago.

Nederhof, A. (1985). Methods of coping with social desirability bias: a review. European Journal of Social Psychology, 15, 263-280.

Nelson, M. R., Brunel, F. F., Supphellen, M., \& Manchanda, R. V. (2006). Effects of Culture, Gender, and Moral Obligations on Responses to Charity Advertising Across Masculine and Feminine Cultures. Journal of Consumer Psychology, 16(1), 45-56.

Nishimura, D. Y., Fath, M., Mullins, R. F., Searby, C., Andrews, M., Davis, R., . . . Sheffield, V. C. (2004). Bbs2-null mice have neurosensory deficits, a defect in social dominance, and retinopathy associated with mislocalization of rhodopsin. Proceedings of the National Academy of Sciences of the United States of America, 101(47), 16588-16593.

Petersen, M. B., Slothuus, R., Stubager, R., \& Togeby, L. (2011). Deservingness versus values in public opinion on welfare: The automaticity of the deservingness heuristic. European Journal of Political Research, 50(1), 24-52. 
Tests of Social Dominance on Charitable Intent Towards Minorities

Pratto, F., Liu, J. H., Levin, S., Sidanius, J., Shih, M., Bachrach, H., \& Hegarty, P. (2000). Social Dominance Orientation and the Legitimization of Inequality Across Cultures. Journal of Cross-Cultural Psychology, 31(3), 369-409.

Pratto, F., Sidanius, J., Stallworth, L. M., \& Malle, B. (1994). Social dominance orientation: A personality variable predicting social and political attitudes. Journal of Personality and Social Psychology, 67(4), 741-763.

Pratto, F., Stallworth, L. M., \& Sidanius, J. (1997). The gender gap: Differences in political attitudes and social dominance orientation. British Journal of Social Psychology, 36(1), 49-68.

Pratto, F., Stewart, A. L., Foels, R., Henkel, K. E., BouZeineddine, F., Laham, S., \& Morselli, D. (2012). Beyond me and mine: The socially-inclusive psychology of people low on social dominance orientation.

Reicher, S. (2004). The Context of Social Identity: Domination, Resistance, and Change. Political Psychology, 25(6), 921-945.

Reiling, H. T. (1958). Federal Taxation: What Is a Charitable Organization? American Bar Association Journal , 525-598.

Rubin, M., \& Hewstone, M. (2004). Social Identity, System Justification, and Social Dominance: Commentary on Reicher, Jost et al., and Sidanius et al. Political Psychology, 25(6), 823-844.

Sellers, J. G., Mehl, M. R., \& Josephs, R. A. (2007). Hormones and personality: Testosterone as a marker of individual differences. Journal of Research in Personality, 41(1), 126-138.

Shamay-Tsoory, S. G., Aharon-Peretz, J., \& Perry, D. (2008). Two systems for empathy: a double dissociation between emotional and cognitive empathy in inferior frontal gyrus versus ventromedial prefrontal lesions. Brain a Journal of Neurology, 132, 617-627.

Sheldon, K. M., \& King, L. (2001). Why positive psychology is necessary. American Psychologist, 56(3), 216. 
Tests of Social Dominance on Charitable Intent Towards Minorities

Sibley, C. G., \& Duckitt, J. (2010). The Ideological Legitimation of the Status Quo: Longitudinal Tests of a Social Dominance Model. Political Psychology, 31(1), 109-137.

Sibley, C. G., \& Liu, J. H. (2004). Attitudes towards Biculturalism in New Zealand: Social Dominance and Pākehā Attitudes towards the General Principles and Resource-Specific Aspects of Bicultural Policy . New Zealand Journal of Psychology, 33(2), 88.

Sibley, C. G., \& Liu, J. H. (2007). New Zealand= bicultural? Implicit and explicit associations between ethnicity and nationhood in the New Zealand context. European Journal of Social Psychology, 37(6), 1222-1243.

Sibley, C. G., \& Wilson, M. S. (2007). Political attitudes and the ideology of equality: Differentiating support for liberal and conservative political parties in New Zealand. New Zealand Journal of Psychology, 36(2), 72-84.

Sibley, C. G., Robertson, A., \& Wilson, M. S. (2006). Social Dominance Orientation and Right-Wing Authoritarianism: Additive and Interactive Effects. Political Psychology, 27(5), 755-768.

Sidanius, J., \& Pratto, F. (1993). The inevitability of oppression and the dynamics of social dominance. In P. Sniderman, \& P. Tetlock (Eds.), Prejudice, Politics, and the American Dilemma (pp. 173-211). Stanford University Press.

Sidanius, J., \& Pratto, F. (1999). Social Dominance: An Intergroup Theory of Social Hierarchy and Oppression. Cambridge: Cambridge University Press.

Sidanius, J., Kteily, N., Sheehy-Skeffington, J., Ho, A. K., Sibley, C., \& Duriez, B. (2013). You're inferior and not worth our concern: The interface between empathy and social dominance orientation. Journal of Personality, 81(3), 313323.

Sidanius, J., Levin, S., Federico, C., \& Pratto, F. (2001). Legitimizing ideologies: The social dominance approach. In J. T. Jost, \& B. Majors (Eds.), The psychology of legitimacy: Emerging perspectives on ideology, justice and intergroup relations (pp. 307-331). New York, Cambridge University Press. 
Tests of Social Dominance on Charitable Intent Towards Minorities

Sidanius, J., Levin, S., Liu, J., \& Pratto, F. (2000). Social dominance orientation, antiegalitarianism and the political psychology of gender: an extension and crosscultural replication. European Journal of Social Psychology, 30(1), 41-67.

Sidanius, J., Liu, J. H., Shaw, J. S., \& Pratto, F. (1994). Social Dominance Orientation, Hierarchy Attenuators and Hierarchy Enhancers: Social Dominance Theory and the Criminal Justice System. Journal of Applied Social Psychology, 24(4), 338366.

Sidanius, J., Pratto, F., \& Bobo, L. (1994). Social dominance orientation and the political psychology of gender: A case of invariance? . Journal of Personality and Social Psychology, 67(6), 998-1011.

Sidanius, J., Pratto, F., \& Mitchell, M. (1993). Ingroup Identification, Social Dominance Orientation, and Differential Intergroup Social Allocation. The Journal of Social Psychology, 134(2), 151-167.

Sidanius, J., Pratto, F., Sinclair, S., \& van Laar, C. (1996). Mother Teresa meets Genghis Khan: The dialectics of hierarchy-enhancing and hierarchy-attenuating career choices. Social Justice Research, 9(2), 145-170.

Smith, J. R., \& McSweeney, A. (2007). Charitable giving: The effectiveness of a revised theory of planned behaviour model in predicting donating intentions and behaviour. Journal of Community \& Applied Social Psychology , 17(5), 363386.

Van Der Linden, S. (2011). Charitable Intent: A Moral or Social Construct? A Revised Theory of Planned Behavior Model . Current Psychology, 30(4), 355-374.

Weisbrot, R. (1990). Freedom bound: A history of America's civil rights movement. EP Dutton.

Wilson, M. S. (2003). Social Dominance and Ethical Ideology: The End Justifies the Means? The Journal of Social Psychology, 143(5), 549-558.

Wilson, M. S., \& Liu, J. H. (2003). Social dominance orientation and gender: The moderating role of gender identity. British Journal of Social Psychology, 42(2), 187-198. 
Tests of Social Dominance on Charitable Intent Towards Minorities

Wilson, M. S., \& Sibley, C. G. (2013). Social Dominance Orientation and Right-Wing Authoritarianism: Additive and Interactive Effects on Political Conservatism. Political Psychology, 34(2), 277-284.

Winterich, K. P., Zhang, Y., \& Mittal, V. (2012). How political identity and charity positioning increase donations: Insights from Moral Foundations Theory. International Journal of Research in Marketing, 29(4), 346-354.

Xu, X. (1998). Asian values revisited: In the context of intercultural news communication. Media Asia, 25, 37-41.

Zahn-Waxler, C., \& Radke-Yarrow, M. (1990). The origins of empathetic concern. Motivation and Emotion, 14(2), 107-130.

Zettler, I., \& Hilbig, B. E. (2010). Attitudes of the selfless: Explaining political orientation with altruism. Personality and Individual Differences, 48, 338-342.

Zettler, I., Hilbig, B. E., \& Haubrich, J. (2011). Altruism at the ballots: Predicting political attitudes and behavior. Journal of Research in Personality, 45(1), 130133.

Zhang, Y. B., Lin, M.-C., Nonaka, A., \& Beom, K. (2005). Harmony, hierarchy and conservatism: A cross-cultural comparison of Confucian values in China, Korea, Japan, and Taiwan. Communication Research Reports , 22(2), 107-115. 
Tests of Social Dominance on Charitable Intent Towards Minorities

\section{Appendix A}

1.) Some groups of people must be kept in their place (SDO_D1)

2.) We should work to give all groups an equal chance to succeed (SDO_E1R)

3.) Group equality should not be our primary goal (SDO_E2)

4.) There's nothing more important than confronting oppression by dominant groups (CDO_1)

5.) Groups at the bottom are just as deserving as groups at the top (SDO_D2R)

6.) We should not push for group equality (SDO_E3)

7.) It's probably a good thing that certain groups are at the top and other groups are at the bottom (SDO_D3)

8.) Less dominant groups must sometimes be willing to follow more dominant groups (CDO_5R)

9.) We should do what we can to equalize conditions for different groups (SDO_E4R)

10.) It is good for weaker groups to rely on more powerful groups (CDO_2R)

11.) No one group should dominate society (SDO_D4R)

12.) We shouldn't try to guarantee that every group has the same quality of life (SDO_E5)

13.) An ideal society requires some groups to be on top and others to be on the bottom (SDO_D5)

14.) Sometimes it's right for a subordinated group to compromise with a dominant group (CDO_3R)

15.) No matter how much effort it takes, we ought to strive to ensure that all groups have the same chance in life (SDO_E6R)

16.) Groups at the bottom should not have to stay in their place (SDO_D6R)

17.) It is unjust to try to make groups equal (SDO_E7)

18.) Some groups of people are simply inferior to other groups (SDO_D7)

19.) Dominant group's influence on subordinated groups is never acceptable (CDO_4)

20.) Group equality should be our ideal (SDO_E8R)

21.) Oppressed groups that resist exploitation by dominant groups are worthy of total respect (CDO_6)

22.) Group dominance is a poor principle (SDO_D8R) 


\section{Appendix B}

1.) Our country desperately needs a mighty leader who will do what has to be done to destroy the radical new ways and sinfulness that are ruining us

2.) Gays and lesbians are just as healthy and moral as anybody else

3.) Our country will be great if we honour the ways of our forefathers, do what the authorities tell us to do, and get rid of the "rotten apples" who are ruining everything

4.) Atheists and others who have rebelled against the established religions are no doubt every bit as good and virtuous as those who attend church regularly

5.) The real key to the "good life" is obedience, discipline, and sticking to the straight and narrow

6.) A lot of our rules regarding modesty and sexual behaviour are just customs which are not necessarily any better or holier than those which other people follow

7.) There are many radical, immoral people in our country today, who are trying to ruin it for their own godless purposes, whom the authorities should put out of action

8.) It is always better to trust the judgment of the proper authorities in government and religion than listen to noisy rabble-rousers in society who are trying to create doubt in people's minds

9.) There is absolutely nothing wrong with nudist camps

10.) There is no "ONE right way" to live life; everybody has to create their own way

11.) Our country will be destroyed someday if we do not smash the perversions eating away at our moral fibre and traditional beliefs

12.) Homosexuals and feminists should be praised for being brave enough to defy "traditional family values"

13.) The situation in our country is getting so serious, the strongest methods would be justified if they eliminated the troublemakers and got us back to our true path

14.) It may be considered old fashioned by some, but having a normal proper appearance is still the mark of a gentleman and, especially, a lady

15.) Everyone should have their own lifestyle, religious beliefs, and sexual preferences, even if it makes them different from everyone else

16.) A "woman's place" should be wherever she wants to be. The days when women were submissive to their husbands and social conventions belong strictly in the past

17.) What our country really needs is a strong, determined leader who will crush evil, and take us back to our true path 
18.) People should pay less attention to the Bible and the other old traditional forms of religious guidance, and instead develop their own personal standards of what is moral and immoral

19.) The only way our country can get through the crisis ahead is to get back to our traditional values, put some tough leaders in power, and silence the troublemakers spreading bad ideas

20.) Our country needs free thinkers who will have the courage to defy traditional ways, even if this upsets many people

21.) There is nothing wrong with premarital sexual intercourse

22.) It would be best for everyone if the proper authorities censored magazines so that people could not get their hands on trashy and disgusting material

23.) It is wonderful that young people today have greater freedom to protest against things they don't like, and to make their own "rules" to govern their behaviour

24.) What our country really needs, instead of more "civil rights," is a good dose of law and order

25.) Some of the best people in our country are those who are challenging our government, criticising religion, and ignoring the "normal way" things are supposed to be done

26.) Obedience and respect for authority are the most important virtues children should learn

27.) Nobody should "stick to the straight and narrow." Instead people should break loose and try out lots of different ideas and experiences

28.) Once our government leaders give us the "go ahead" it will be the duty of every patriotic citizen to help stomp out the rot that is poisoning our country from within

29.) We should treat protestors and radicals with open arms and open minds, since new ideas are the lifeblood of progressive change

30.) The facts on crime, sexual immorality, and the recent public disorders all show we have to crack down harder on deviant groups and troublemakers if we are going to save our moral standards and preserve law and order 


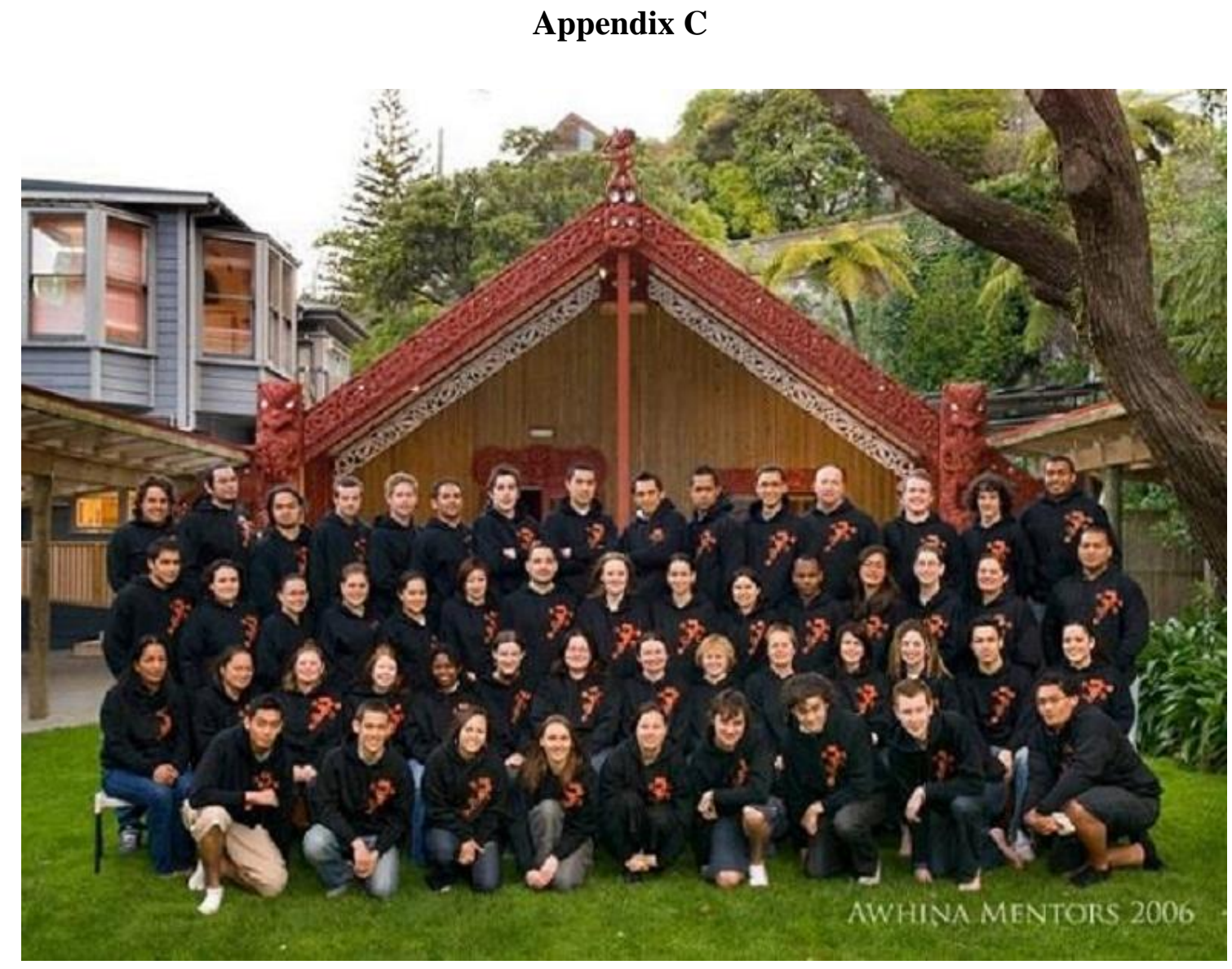

University study is now an option for more New Zealanders than ever before. This also means that people coming to university come with very different experiences, skills, and understandings of what it means to be a student. Additionally, study is just one part of people's lives, and sometimes people need a little support to help them achieve the best results that they can.

For these reasons, universities typically offer a range of services, organisations, and opportunities to work alongside students. Typically, this might involve assigning a mentor for new students, chances to meet with people to work through what is expected in assignments, advice on foundation skills necessary to succeed at university, or a place to study and putting students in contact with others like them.

At Victoria University, examples of these types of organisations include Te Rōpū Āwhina, and Te Pūtahi Atawhai, that provide opportunities for Māori and Pasifika students to learn to succeed. 


\section{Appendix D}

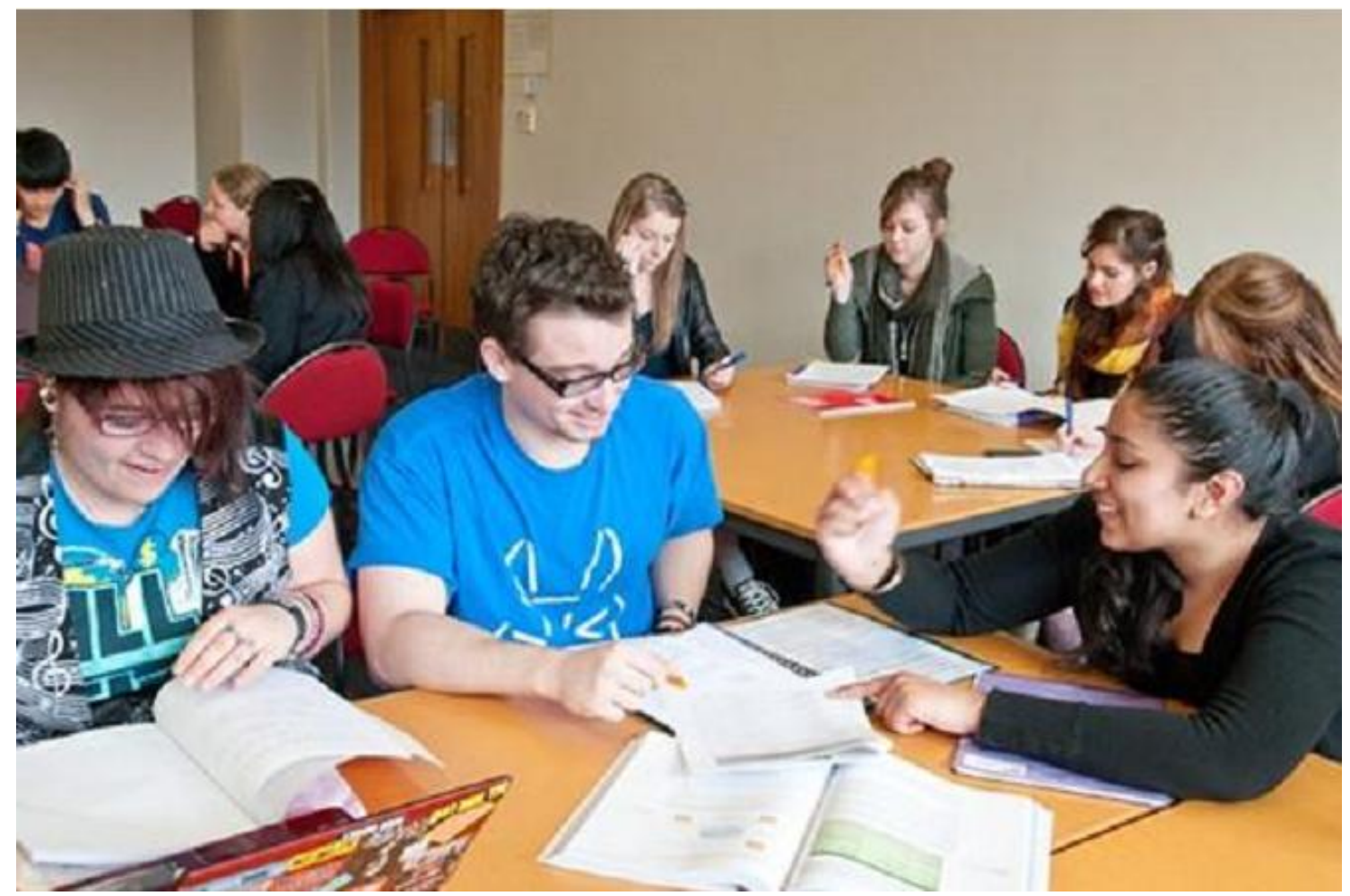

University study is now an option for more New Zealanders than ever before. This also means that people coming to university come with very different experiences, skills, and understandings of what it means to be a student. Additionally, study is just one part of people's lives, and sometimes people need a little support to help them achieve the best results that they can.

For these reasons, universities typically offer a range of services, organisations, and opportunities to work alongside students. Typically, this might involve assigning a mentor for new students, chances to meet with people to work through what is expected in assignments, advice on foundation skills necessary to succeed at university, or a place to study and putting students in contact with others like them.

At Victoria University, examples of these types of organisations include Student Learning, and Peer Assisted Study Support (PASS), that provide opportunities for students to learn to succeed. 
Appendix E

This question asks about the effectiveness of student services

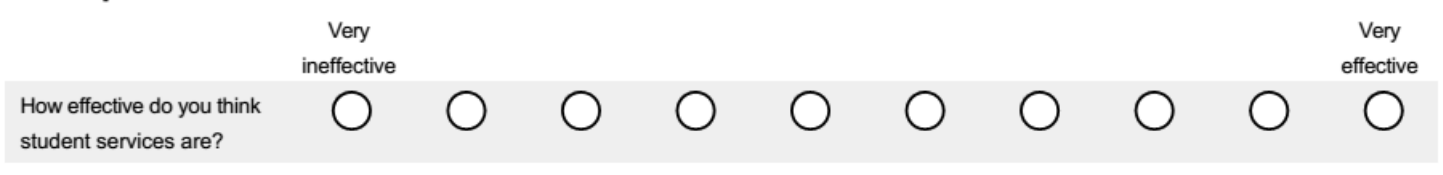

This question asks about the IMPORTANCE of student services

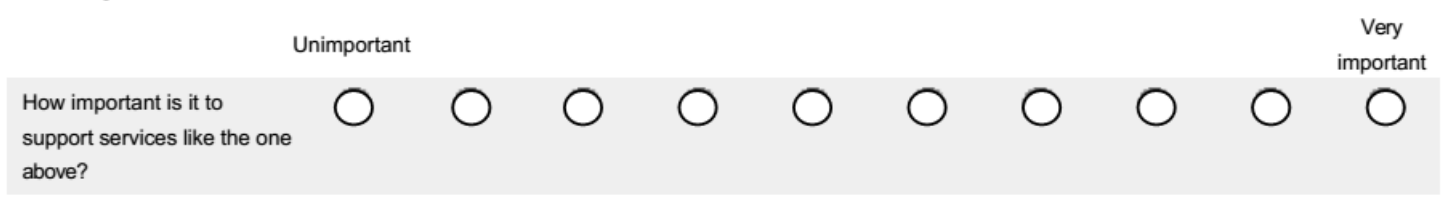

This question asks about the effectiveness of the advert ABOVE

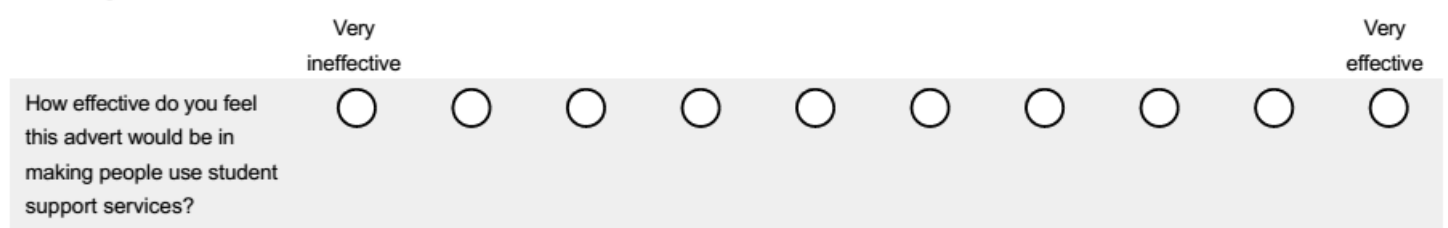

Please indicate what percentage of students you think use student support services. 
Tests of Social Dominance on Charitable Intent Towards Minorities

\section{Appendix F}

Imagine a hypothetical scenario in which, in addition to IPRP credit, you also received $\$ 5$ for your participation in this study.

Imagine that you could either keep the $\$ 5$, or donate a portion of it into a public pool of money. Your fellow participants would have the same opportunity. Any money in the public pool would be doubled, and then distributed evenly across the participants as well as Te Rōpū Âwhina. You and the other participants would not be allowed to discuss it in any way until after the money has been handed out.

In this hypothetical scenario, how much money would you put into the public pool? Amount donated: 
Tests of Social Dominance on Charitable Intent Towards Minorities

\section{Appendix G}

Imagine a hypothetical scenario in which, in addition to IPRP credit, you also received $\$ 5$ for your participation in this study.

Imagine that you could either keep the $\$ 5$, or donate a portion of it into a public pool of money. Your fellow participants would have the same opportunity. Any money in the public pool would be doubled, and then distributed evenly across the participants as well as Student Learning Support. You and the other participants would not be allowed to discuss it in any way until after the money has been handed out.

In this hypothetical scenario, how much money would you put into the public pool? Amount donated: 